\title{
La diaspora kurde en Suède. Conservation, production et diffusion d'un savoir linguistique
}

The Kurdish diaspora in Sweden. Conservation, production and dissemination of linguistic knowledge

\section{Clémence Scalbert-Yücel}

\section{OpenEdition \\ Journals}

\section{Édition électronique}

URL : https://journals.openedition.org/ejts/771

DOI : 10.4000/ejts. 771

ISSN : $1773-0546$

Éditeur

EJTS

\section{Référence électronique}

Clémence Scalbert-Yücel, « La diaspora kurde en Suède. Conservation, production et diffusion d'un savoir linguistique », European Journal of Turkish Studies [En ligne], 5 | 2006, mis en ligne le 05 mars 2015, consulté le 10 décembre 2021. URL : http://journals.openedition.org/ejts/771 ; DOI : https:// doi.org/10.4000/ejts. 771 


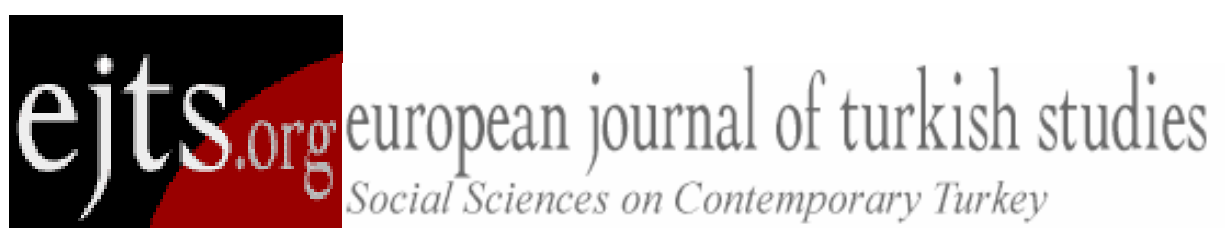

Citation: Scalbert Yücel, Clémence (2006) 'La diaspora kurde en Suède. Conservation, production et diffusion d'un savoir linguistique', European Journal of Turkish Studies, Thematic Issue N5, Power, ideology, knowledge - deconstructing Kurdish Studies, URL : http://www.ejts.org/document771.html

To quote a passage, use paragraph $(\S)$

\title{
La diaspora kurde en Suède. Conservation, production et diffusion d'un savoir linguistique
}

Clémence Scalbert Yücel

\begin{abstract}
The Kurdish diaspora has organised itself quite quickly, especially since the 1980 Coup in Turkey. Although the diasporic space may in general be described as decentralized, Sweden appears to take a major part in the field of linguistic and literary activities. This is due both to the commitment of the migrants to the Kurdish cause and to the migration and cultural policies of the Swedish state. On the one hand, the migration policies enable migrant population to learn their mother tongue, considered as the vehicle of their culture. Education in Kurdish language has thus developed and become institutionalised in the host country since the beginning of the 1980s. On the other hand, cultural policies allocate grant and funding to cultural institutions and writers, which then contributes to the development of strong Kurdish archiving editorial and writing activities. Since the beginning of the 1990s, the relationships between the migrants in Sweden and the homeland have grown more intense, highlighting the diasporic organisation of the community. However, with the legislation reforms, new possibilities granted to Kurdish publishers and writers in Turkey may transform the role of the Kurdish diaspora in Sweden.
\end{abstract}


Citation: Scalbert Yücel, Clémence (2006) 'La diaspora kurde en Suède. Conservation, production et diffusion d'un savoir linguistique', European Journal of Turkish Studies, Thematic Issue №5, Power, ideology, knowledge - deconstructing Kurdish Studies, URL : http://www.ejts.org/document771.html

To quote a passage, use paragraph (§)

Alors que la pratique orale comme écrite de la langue ainsi que l'expression même d'une identité kurde sont interdites et réprimées en Turquie jusqu'au début des années 1990, la diaspora kurde ${ }^{1}$ qui se structure très rapidement dès les années 1980 semble jouer un rôle extrêmement fort - voire majeur - dans les activités de production identitaire et notamment linguistique. La diaspora a souvent été définie par une absence de logique bipolaire entre un lieu de départ et un lieu d'arrivée (Simon 1995 : 15, 216) et surtout par son caractère extrêmement 'décentralisé' et 'polycentrique' (Bruneau 1995: 16; MaMung). II nous semble toutefois que certains espaces la polarisent nettement et parmi eux, d'abord, le pays d'origine, autour duquel la construction identitaire prend sens et forme. En outre, dans l'espace diasporique réticulé, une spécialisation peut se développer dans certains lieux, du fait des caractéristiques d'un pays d'accueil ou de la population migrante.

[2] Notre choix de travailler sur la section suédoise de la diaspora kurde s'explique par cette distinction fonctionnelle et par une hiérarchisation des espaces diasporiques. En effet, dans l'espace diasporique kurde, dès le début des années 1980, la Suède se construit nettement comme le refuge de la langue-identité et de sa mémoire mais aussi comme l'espace de la création linguistique et littéraire kurde. Elle est en ce sens lieu de production d'un savoir spécifique : un savoir sur sa langue mais aussi un savoir sur soi, sur l'identité. Elle produit ce savoir, le conserve et le diffuse. II s'agira donc ici de définir le rôle particulier de la Suède en matière de production identitaire - et essentiellement linguistique -, de comprendre comment la Suède a acquis ce rôle et de s'interroger sur la maintenance de ce rôle.

[3] La nature de l'immigration kurde en Suède mais surtout les politiques d'immigration ainsi que les politiques culturelles suédoises permettent l'émergence d'un pôle diasporique kurde, caractérisé d'une part par ses activités de production et de conservation de l'identité et de la langue kurde - conçue, par les acteurs de la diaspora, comme un marqueur essentiel de la kurdicité -, d'autre part par ses activités de création en matière éditoriale et littéraire. Si ce pôle fonctionne d'abord en cercle fermé (autour de la Suède et, dans une moindre mesure, autour des autres lieux européens de la diaspora), nous verrons qu'il joue un rôle extrêmement important en Turquie dès lors que

\footnotetext{
${ }^{1}$ Nous tiendrons essentiellement compte de la population kurde (originaire) de Turquie et de sa langue le kurmandji (le zazaki se trouvant clairement dans une situation de langue minoritaire parmi les langues kurdes). En effet, les évolutions politiques dans chaque pays étant très différentes, la situation de la langue kurde, les relations entre les populations kurdes et leur langue changent d'un pays à l'autre.
} 
Citation: Scalbert Yücel, Clémence (2006) 'La diaspora kurde en Suède. Conservation, production et diffusion d'un savoir linguistique', European Journal of Turkish Studies, Thematic Issue №5, Power, ideology, knowledge - deconstructing Kurdish Studies, URL : http://www.ejts.org/document771.html

To quote a passage, use paragraph (§)

l'intervention dans ce pays est rendue possible dans les années 1990 à un moment où l'emploi du kurde se libéralise progressivement. Pourtant cette libéralisation semble mettre en cause la pérennité du rôle de la diaspora kurde en Suède.

\section{La construction d'une spécificité suédoise}

\section{Une immigration kurde fortement politisée}

[4] L'immigration kurde vers la Suède commence progressivement dès les années 1960 et s'intensifie - relativement - après les coups d'État de 1971 et surtout de 1980. Elle reste toutefois assez peu dense par rapport aux mouvements migratoires vers l'Allemagne ou même vers la France ${ }^{2}$. Cette immigration kurde vers la Suède conjugue motifs politiques et économiques. Selon Rohat Alakom, la migration économique est la plus ancienne. Elle touche d'abord les Kurdes d'Anatolie centrale (régions d'Aksaray et de Konya). Ceux-ci arrivent comme travailleurs dans les régions de Stockholm et de Göteborg dès les années 1960 (Alakom 1991: 12; Alakom 2002: 39). Toutefois, ces Kurdes d'Anatolie centrale sont aussi partis du fait d'activités politiques au pays. Et, en exil, beaucoup d'entre eux ont redécouvert leur kurdicité puis travaillé à sa conservation. Ceci est attesté dans les pages de Birnebûn, revue publiée par les Kurdes d'Anatolie (éditions Apec) depuis 1997. Son titre, 'Ne pas oublier', est très significatif de son objectif. II est ainsi important de relativiser la distinction souvent faite entre migration politique et économique. Stéphane Dufoix note en effet : 'outre le fait qu'il est souvent très difficile de faire la part des choses entre les causes économiques et politiques de la migration, cette vision empêche de prendre en compte les 'changements de nature' dans les pays d'accueil: 'migrants économiques' qui se politisent, 'migrants politiques' qui n'interviennent pas dans la mise en place d'activités politiques en direction du pays d'origine, réactivations de liens avec le pays de la part de gens qui en semblaient détachés...' (Dufoix 2003 : 70).

[5] Les immigrants kurdes en Suède sont toutefois, souvent, des individus très politisés : ils sont pour beaucoup militants des nombreux partis politiques que le coup

${ }^{2}$ S'il n'existe aucun recensement fiable concernant la population kurde en Europe, nous pouvons dire qu'il existe 500 à 600000 Kurdes en Allemagne, 120 à 130000 en France et 20 à 30000 en Suède. Voir http://www.institutkurde.org/kurdoramal. Consulté le 22/06/2007. 
Citation: Scalbert Yücel, Clémence (2006) 'La diaspora kurde en Suède. Conservation, production et diffusion d'un savoir linguistique', European Journal of Turkish Studies, Thematic Issue №5, Power, ideology, knowledge - deconstructing Kurdish Studies, URL : http://www.ejts.org/document771.html

To quote a passage, use paragraph (§)

d'État, a brisés en Turquie, mais qui connaissent un nouvel essor en Suède comme ailleurs en Europe ${ }^{3}$. Cette immigration est enfin celle d'hommes de lettres tels que Rojen Barnas, Mehmet Emin Bozarslan, Cigerxwîn ${ }^{4}$, qui étaient écrivains avant leur arrivée en Suède dans les années 1980. II est nécessaire de noter que la distinction entre militant et homme de lettre est, elle aussi, difficile à effectuer. En effet, les militants politiques exilés en Suède se tournent vers une nouvelle forme de militantisme. Pour beaucoup, l'activité strictement politique semble vaine à l'extérieur du pays. On remarque donc un changement d'orientation, de l'activisme politique à l'activisme culturel. Mais la culture est, elle aussi, conçue comme un engagement. L'écrivain kurde le plus connu, Mehmet Uzun, utilise le terme 'd'engagement' : si la langue n'avait pas été en 'danger de disparition', dit-il, en Suède, il aurait certainement écrit en suédois. Mais, ajoute-il, en écrivant en kurde, il s'engageait (Uzun 1996: 118-119). Cette forme nouvelle de militantisme culturel, vigoureux en Suède, est en outre, nous le verrons, encouragée par les politiques suédoises.

[6] La population kurde immigrée en Suède s'organise très rapidement en associations culturelles, politiques ou régionales. La première organisation kurde à voir le jour est une association de travailleurs dont la plupart des fondateurs sont des Kurdes d'Anatolie centrale. II s'agit de l'Association des Travailleurs Kurdes - Suédois (Komeleya Karkeran a Kurdî - Swedî), fondée en 1976 (Alakom 2002 : 40). Très vite, un dense réseau associatif se tisse dans tout le territoire, réseau qui est chapeauté, dès 1981, par la Fédération des Associations du Kurdistan (Federasyona Komelên Kurdistanê) ${ }^{5}$. Cette Fédération regroupe toutes les associations qui veulent bien s'y joindre, sans distinction politique, linguistique ou régionale. Elle rassemble aujourd'hui une quarantaine d'organisations réparties dans toute la Suède. Elle publie depuis 1982 une revue, Berbang, qui comporte une partie en kurmandji, une autre en zazaki et une dernière en sorani. Un

\footnotetext{
${ }^{3}$ Les partis vont s'y réorganiser ainsi que les publications de ces partis. En revanche, c'est en Allemagne que ces partis seront les plus vigoureux, certainement car on y trouve une population kurde mobilisable bien plus importante qu'en Suède.

${ }^{4}$ Le cas de Cigerxwîn est particulier. Si c'est un Kurde de Syrie, dès ses premières expériences politicolittéraires (avec Hawar notamment dans les années 1930-1940) il fut très proche des Kurdes de Turquie et se retrouve à les côtoyer en Suède.

${ }^{5}$ Elle sera par la suite renommée Fédération des associations kurdes en Suède (Federasyona Komelên Kurd li Swedê).
} 
Citation: Scalbert Yücel, Clémence (2006) 'La diaspora kurde en Suède. Conservation, production et diffusion d'un savoir linguistique', European Journal of Turkish Studies, Thematic Issue N5, Power, ideology, knowledge - deconstructing Kurdish Studies, URL : http://www.ejts.org/document771.html

To quote a passage, use paragraph (§)

des objectifs incontestables de la Fédération est de rassembler, dans la diversité, les Kurdes de toute origine et de toute langue ${ }^{6}$.

[7] On remarque que l'intégration est extrêmement rapide en Suède: quelques années suffisent à la création de la Fédération et à ses activités de lobbying ; très vite, les Kurdes s'affichent également sur les listes électorales suédoises ${ }^{7}$. La nature fortement politisée des migrants kurde en Suède (présence de mouvements politiques ou de personnes déjà mobilisées en Turquie) explique en partie cette intégration. Cette capacité à se mobiliser dans le pays d'accueil autour du pays d'origine marque véritablement le fonctionnement diasporique du groupe. La constitution de la diaspora ne se fait qu'avec l'apparition d'une élite intellectuelle mais aussi financière qui naît progressivement à l'extérieur du pays d'origine et qui prend en charge l'organisation de la communauté (Bruneau 1995 : 9). Cette intégration toutefois est certainement aussi en grande partie due aux politiques migratoires suédoises. Elles permettent l'intégration de la communauté kurde en Suède qui, à son tour, se mobilise afin de tirer profit de ces politiques. Ainsi, la capacité de la diaspora kurde à fonctionner en tant que telle mais surtout l'essor de la Suède en tant que pôle diasporique dans le domaine linguistique et culturel s'expliquent essentiellement par les politiques d'immigration et par les politiques culturelles suédoises, exceptionnelles en Europe.

\section{Des politiques migratoires inédites en Europe}

[8] La Suède, traditionnel pays d'émigration, devient, dès les années 1960, un pays d'immigration. Déjà, dans les années 1970, elle connaît une immigration importante. L'État suédois constatant que l'immigration ne cesserait pas et que beaucoup d'immigrés ne rentreraient plus chez eux, le Parlement adopte, en 1975, trois principes sur lesquels

\footnotetext{
${ }^{6}$ Ainsi, selon une enquête réalisée par la Fédération (et publiée dans Berbang 78, 1992, pp. 6-7), les lecteurs de la revue originaires de Turquie sont quasiment aussi nombreux que ceux originaires d'Irak (ils forment à eux deux $74 \%$ des lecteurs).

7 La population kurde en Suède est particulièrement bien intégrée à la vie politique, en particulier, locale (participation active aux partis, activités au sein du parlement, des mairies, etc.). Nalîn Baksî, sœur de l'écrivain Mahmut Baksî, est élue députée suédoise en 1994 (Parti social-démocrate). De nombreux Kurdes sont actifs politiquement, en particulier au sein de ce parti, longtemps dominant dans la vie politique suédoise. En 1997, Keya Îzol, président de la Fédération, invite les Kurdes à voter aux élections législatives et soutient fortement les candidats kurdes présentés dans Berbang ( ${ }^{\circ}$ spécial, septembre 1997). Selon la revue, l'intérêt d'avoir des représentants kurdes est double : d'une part, cela peut améliorer la situation des Kurdes en Suède (et des étrangers en général). D'autre part, cela peut aider à renforcer la place de la question kurde dans l'actualité.
} 
Citation: Scalbert Yücel, Clémence (2006) 'La diaspora kurde en Suède. Conservation, production et diffusion d'un savoir linguistique', European Journal of Turkish Studies, Thematic Issue №5, Power, ideology, knowledge - deconstructing Kurdish Studies, URL : http://www.ejts.org/document771.html

To quote a passage, use paragraph (§)

devait désormais s'appuyer la politique d'immigration: égalité, liberté de choix et coopération ${ }^{8}$. Par égalité, il est entendu que les droits et les opportunités doivent être les mêmes pour les populations immigrées que pour le reste de la population. Tous les membres de la société doivent bénéficier des mêmes possibilités de conserver et de développer leur langue ainsi que d'exercer des activités ayant trait à leur culture d'origine. Par liberté de choix, il est entendu que la population immigrée doit elle-même décider de son degré d'identification à la culture suédoise, du degré de conservation de sa culture d'origine ; quant aux Suédois, ils se doivent de respecter ce choix et le refus possible de l'assimilation. Les immigrés doivent pouvoir participer pleinement à la vie sociale et politique suédoise; c'est ce que l'on entend par coopération?.

[9] Certains chercheurs remarquent alors les problèmes linguistiques qui touchent les communautés immigrées et d'abord finlandaises. On parle de 'semi-linguisme' (Halvspråkighet) pour caractériser la situation dans laquelle se trouvent les enfants finnois ne parlant et n'écrivant correctement ni leur langue d'origine ni la langue suédoise. II est relevé que cette situation aurait des conséquences néfastes pour le développement psychologique et émotionnel de l'enfant. Le terme de 'semi-linguisme' est ensuite repris pour caractériser la situation des immigrés d'origines diverses (Cabau 1996 : 332).

[10] L'idée de 'bilinguisme actif' est alors développée dès 1974 dans un rapport sur la situation des minorités et des immigrants (Sou 1974). Elle est prônée dans la proposition gouvernementale de 1975 sur la politique envers les immigrés et les minorités ${ }^{10}$. Le 'bilinguisme actif' correspondrait à la situation dans laquelle pourrait se trouver un individu qui parlerait et écrirait couramment deux langues; chaque langue est fortement inscrite dans sa culture et est aussi 'véhicule' de 'sa' culture. Un 'bilingue actif pourrait évoluer en étant à l'aise dans les deux environnements culturels via les langues. L'idée est alors avancée de promouvoir les langues maternelles (modersmån), à l'époque plus volontiers appelées par les Suédois 'langues domestiques' (hemspråk) faisant référence à la langue (vivante) qui constitue un phénomène réel dans le foyer de l'enfant immigré (in Cabau-

8 Voir Regeringsproposition (1975) 26 om riktlinjer för invandrar - och minoritetspolitikers. Cité par CambauLampa 1998.

9 Pour un travail détaillé concernant les politiques migratoires suédoises et l'enseignement des langues des immigrés voir Cabau 1996. Cet ouvrage est une thèse de doctorat, publiée en 1998. N'ayant pu avoir accès à l'ouvrage publié nous nous servons ici de la thèse.

10 Voir Regeringsproposition (1975) 26 om riktlinjer för invandrar - och minoritetspolitikers. Cette proposition est citée par Cabau-Lampa 1999. 
Citation: Scalbert Yücel, Clémence (2006) 'La diaspora kurde en Suède. Conservation, production et diffusion d'un savoir linguistique', European Journal of Turkish Studies, Thematic Issue №5, Power, ideology, knowledge - deconstructing Kurdish Studies, URL : http://www.ejts.org/document771.html

To quote a passage, use paragraph (§)

Lampa 1999 : 77). La langue est mise en relation avec le foyer, les parents ; elle constitue un élément intrinsèque, indissociable de la culture. D'où le terme de 'langue-culture d'origine' (LCO) que choisit d'utiliser Béatrice Cabau (1996), et que nous reprenons ici. En 1985, la définition de la langue domestique est réduite à une langue de communication quotidienne au sein du foyer (Cabau-Lampa 1999 : 77). A partir de 1997, la législation n'usera plus du terme de langue domestique mais optera pour celui de langue maternelle (Boyd 2001: 188-190)11.

[11] S'appuyant sur l'idée du 'bilinguisme actif', la réforme de 1977 sur les LCO rend possible leur enseignement ${ }^{12}$. Tous les élèves qui ont une langue maternelle autre que le suédois - et qui en font usage au sein de leur foyer - peuvent suivre des cours de LCO tout au long de la période de scolarité obligatoire (classes 1 à 9 du primaire et du secondaire; élèves âgés de 7 à 16 ans). Ces cours doivent servir à acquérir des connaissances linguistiques approfondies : connaître la grammaire, la structure de la langue, etc. La langue étant considérée comme un élément intrinsèque de la culture, cet enseignement vise également à développer des connaissances sur le pays et la culture d'origine. Cet enseignement vise le renforcement de l'estime de soi mais aussi le développement d'individus bilingues avec des compétences et des identités culturelles duales $^{13}$. Cet enseignement est ainsi tout à fait propice au travail de construction et de transmission de la mémoire, qui peut devenir, selon la façon dont il est mené, un lieu véritable de développement des diasporas. Intégrée à la société d'accueil, la communauté diasporique crée sa mémoire, (re)construit son passé collectif et les transmet, malgré l'éloignement au pays d'origine.

[12] Le système d'enseignement des LCO a été remanié à plusieurs reprises depuis 1977. Aujourd'hui, les autorités municipales prennent l'ensemble des dispositions concernant les écoles et sont relativement libres d'organiser l'enseignement à leur guise. Elles sont dues de fournir un enseignement de langues domestiques à tout groupe de cinq élèves ou plus et si des enseignants sont disponibles ${ }^{14}$. Plusieurs écoles peuvent se réunir

11 L'expression 'langue domestique' a été critiquée dans les années 1990. On a jugé qu'elle pouvait déprécier une langue qui serait perçue comme n'étant parlée qu'au sein de l'espace privé (Cambau-Lampa 1998 : 77-78).

12 Regeringsproposition (1975-1976) 118 om hemspråksundervisning för invandrarbarb.

${ }^{13} \mathrm{http://www.skolverket.se/sb/d/354/a/1256.} \mathrm{Site} \mathrm{consulté} \mathrm{le} \mathrm{25/06/2007.}$

14 Depuis 1998, les langues sami, finnoise, meänkieli (finnois parlé au nord de la Suède), yiddish et romanichib se sont vu accorder le statut de langues minoritaires selon les critères de l'Union européenne. 
Citation: Scalbert Yücel, Clémence (2006) 'La diaspora kurde en Suède. Conservation, production et diffusion d'un savoir linguistique', European Journal of Turkish Studies, Thematic Issue №5, Power, ideology, knowledge - deconstructing Kurdish Studies, URL : http://www.ejts.org/document771.html

To quote a passage, use paragraph (§)

afin d'organiser ces cours. Cet enseignement est toujours optionnel et il y a différentes manières de le dispenser; dans tous les cas, chaque élève reçoit deux à trois heures d'enseignement par semaine. L'élève peut choisir sa langue maternelle en tant qu'option tout au long de sa scolarité obligatoire (classe 1 à 9) ; dans le secondaire (classes 6 à 9), l'élève peut choisir d'étudier sa langue maternelle à la place d'une langue étrangère ; enfin, l'élève peut étudier sa langue maternelle dans des horaires laissés libres par l'emploi du temps. Dans les trois dernières années du secondaire (non obligatoires), l'élève peut également poursuivre l'apprentissage de sa langue ${ }^{15}$. Dans les écoles où un profil linguistique spécifique apparaît, l'enseignement de certaines matières peut être dispensé en $\mathrm{LCO}^{16}$. Ces enfants dont la langue maternelle n'est pas le Suédois peuvent, de manière parallèle, choisir une matière intitulée 'Suédois comme langue secondaire'. Cette matière doit aider les élèves à développer les compétences nécessaires pour suivre l'ensemble du cursus en suédois. Cet enseignement est disponible pendant toute la scolarité primaire et secondaire. Environ 60 langues sont enseignées aujourd'hui en tant que langues maternelles en Suède; le Kurde figure parmi les dix premières (Cabau-Lampa 1999 : 81 82 ; Boyd 2001 : 181).

\section{L'enseignement de la langue kurde en Suède}

\section{Une formation supérieure pour les enseignants}

[13] Selon Rohat Alakom (2006 : 183), l'enseignement de la langue kurde en tant que LCO commence dès l'adoption de la réforme en 1977 dans le quartier de Hudding à Stockholm. Ses débuts sont très peu effectifs du fait, d'une part, du faible nombre d'enfants

Contrairement aux langues des immigrés, les membres des cinq minorités peuvent suivre un enseignement en leur langue même s'ils constituent un groupe inférieur à cinq élèves au sein de l'école et même s'ils ne pratiquent pas leur langue maternelle à la maison. Voir http://modersmal.skolutveckling.se/projekt/modules.php?op=modload\&name=Sections\&file=index\&req=view article\&artid=11\&page=1 et http://www.skolverket.se/sb/d/354/a/1256. Sites consultés le 25/06/2007.

$15 \quad$ Voir

http://modersmal.skolutveckling.se/projekt/modules.php?op=modload\&name=Sections\&file=index\&req=view article\&artid=11\&page=1, site consulté le 25/06/2007 ; voir aussi Cabau-Lampa 1999 : 80-81.

16 Voir http://www.skolverket.se/sb/d/354/a/1256. Site consulté le 25/06/2007. Ceci existait déjà en 1985. Comme l'atteste Rexnegir, les locuteurs de grandes langues (à l'époque le finnois, le grec, le yougoslave et, dans certains lieux, le turc et l'assyrien) peuvent bénéficier d'un enseignement en LCO dans les six premières années de l'école obligatoire. Rexnegir, Z. (1985) 'Li Swêdê Perwerde û Hindekariya Kurdî' [Éducation et enseignement du kurde en Suède], Berbang 4, p. 5. 
Citation: Scalbert Yücel, Clémence (2006) 'La diaspora kurde en Suède. Conservation, production et diffusion d'un savoir linguistique', European Journal of Turkish Studies, Thematic Issue N5, Power, ideology, knowledge - deconstructing Kurdish Studies, URL : http://www.ejts.org/document771.html

To quote a passage, use paragraph (§)

kurdes présents dans le pays, d'autre part, de l'absence de matériel éducatif et de professeurs kurdes. Selon Reşo Zîlan, ces professeurs étaient très rarement formés, et, puisqu'ils provenaient de Turquie, leur connaissance du kurde était très limitée: ils possédaient les bases orales mais n'avaient aucune connaissance en grammaire. Par ailleurs, aucune association ne pouvait alors exprimer les souhaits de la population kurde auprès de l'État suédois. L'action de la Fédération des Associations du Kurdistan nouvellement créée aurait ainsi joué un rôle crucial dans l'ouverture de ces classes ${ }^{17}$.

[14] Suite à une requête de la Fédération, auprès du Ministère des Universités et de l'Enseignement Supérieur, une section de kurde est ouverte à l'École Normale de Stockholm (Högskolan för Lärarutbildning i Stockholm). Les étudiants de l'École normale suivent des cours de pédagogie, de langues, de littérature et d'histoire ${ }^{18}$. Ils perfectionnent le sorani comme le kurmandji et apprennent les trois alphabets utilisés par les Kurdes (latin, arabe, cyrillique). La première promotion, en 1984, est constituée de vingt deux personnes dont dix-neuf étaient originaires du Kurdistan de Turquie ${ }^{19}$. Dès 1984, la Fédération, fait un important battage médiatique afin de mobiliser des personnes susceptibles de devenir enseignants. Aujourd'hui, il y aurait environ 200 enseignants de kurde en tant que LCO en Suède (Alakom 2006 : 188).

Les élèves et leurs langues

$\begin{array}{llll}\text { Années } & & \text { Kurmandji } & \text { Sorani } \\ 1997 & \text { Ayant droit } & 3441 & 996 \\ & \text { Participants } & 1861 & 693 \\ & & 54 \% & 69,5 \% \\ 1998 & \text { Ayant droit } & 3904 & 1237 \\ & \text { Participants } & 2159 & 835 \\ & & 55 \% & 67,5 \% \\ 1999 & \text { Ayant droit } & 3864 & 1888\end{array}$

17 'Li Stockolmê di Xwendegeha Bilind a Mamostetiyê de Beşa Kurdî Vebû' [Une section de kurde a été ouverte à l'École Normale de Stockholm], Armanc 52, 1984, p 12-13. Reşo Zîlan est un des deux premiers professeurs à y enseigner le kurde.

18 Pour un aperçu général de la formation des enseignants de langue maternelle aujourd'hui, on se référera à Cabau-Lampa 1999 : 78-79.

19 'Li Stockolmê di Xwendegeha Bilind a Mamostetiyê de Beşa Kurdî Vebû' [Une section de kurde a été ouverte à l'École Normale de Stockholm], Armanc 52, 1984, p. 12. 
Citation: Scalbert Yücel, Clémence (2006) 'La diaspora kurde en Suède. Conservation, production et diffusion d'un savoir linguistique', European Journal of Turkish Studies, Thematic Issue N5, Power, ideology, knowledge - deconstructing Kurdish Studies, URL : http://www.ejts.org/document771.html

To quote a passage, use paragraph $(\S)$

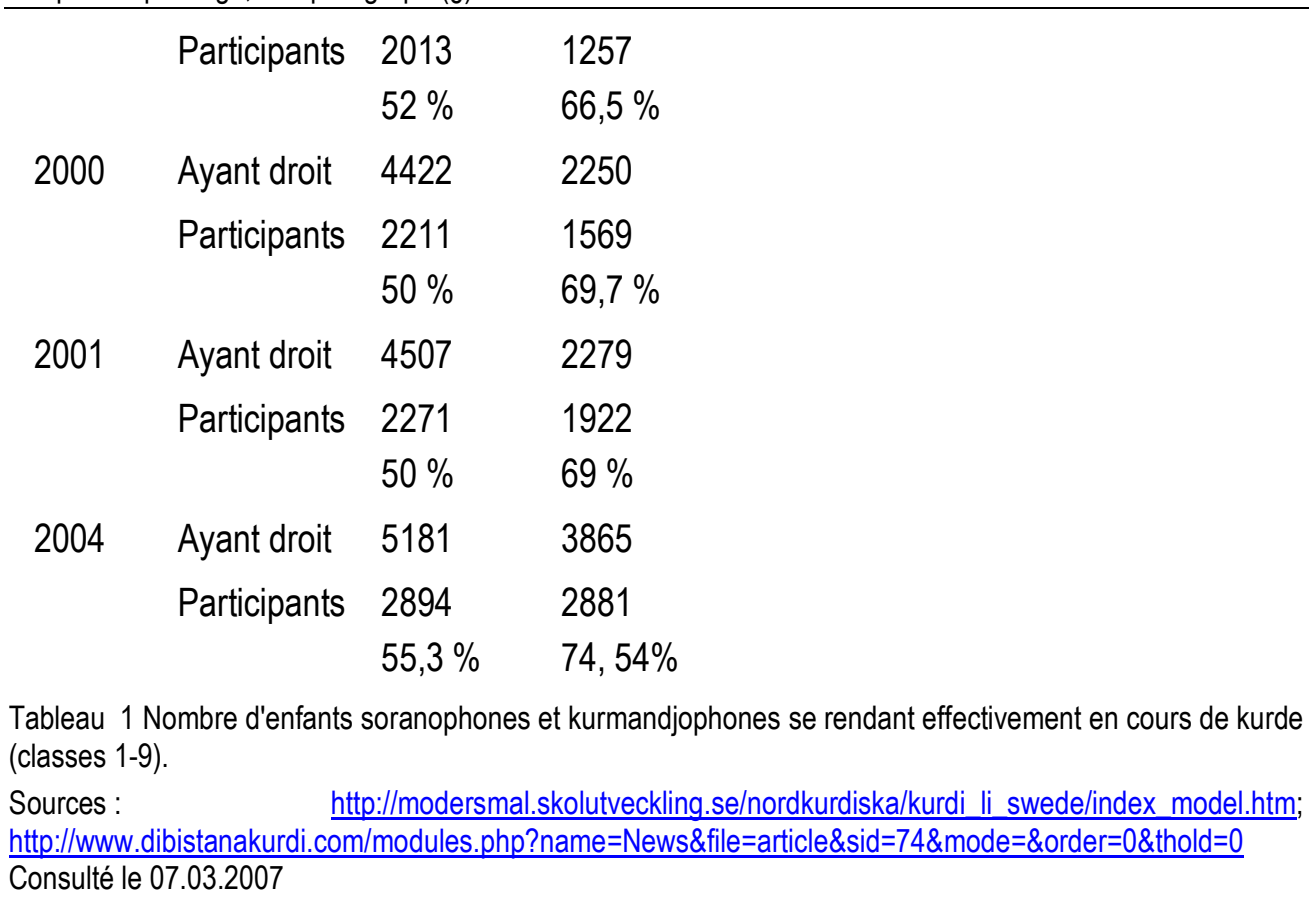

[15] Dans l'enseignement primaire et secondaire, le nombre d'élèves kurdes étudiant leur langue maternelle va croissant. Aujourd'hui, on compte plus de 5000 élèves apprenant le sorani ou le kurmandji en tant que LCO. A la lecture du tableau 1, on constate aisément que le chiffre en valeur brute des élèves ayant droit à ces cours comme celui des élèves y participant effectivement, est toujours croissant, pour les soranophones comme pour les kurmandjophones. Toutefois, en valeur relative, on observe une baisse chez les kurmandjophones et une certaine stabilité chez les soranophones. En outre, l'écart est considérable entre la proportion de soranophones et de kurmandjophones se rendant effectivement en classe de langue maternelle : $50 \%$ seulement des ayant droit kurmandjophones (dont la plupart provient de Turquie) profitent de leur droit contre près de $70 \%$ des soranophones (originaires d'Iran et Irak). Haydar Diljen, enseignant de kurde, explique ce fait par deux facteurs : d'une part, l'assimilation dont les Kurdes de Turquie ont été victimes; d'autre part, la forte et quasiment exclusive utilisation de la langue kurde dans tous les domaines de la vie politique, sociale et culturelle au Kurdistan d'Irak ${ }^{20}$. En effet, les Kurdes irakiens utilisent quotidiennement la langue kurde, dans tous les domaines, ce qui est plus rare chez les Kurdes de Turquie qui ont acquis la langue dominante - le turc.

20 http://www.dibistanakurdi.com/modules.php?name=Content\&pa=showpage\&pid=21. Consulté le 07.03.2007. 
Citation: Scalbert Yücel, Clémence (2006) 'La diaspora kurde en Suède. Conservation, production et diffusion d'un savoir linguistique', European Journal of Turkish Studies, Thematic Issue №5, Power, ideology, knowledge - deconstructing Kurdish Studies, URL : http://www.ejts.org/document771.html

To quote a passage, use paragraph (§)

[16] Ce déséquilibre est significatif et se retrouve, de manière inversée, dans des domaines proches de l'enseignement, comme l'édition. Les principaux activistes kurdes en Suède, dans le domaine linguistique et littéraire, sont originaires de Turquie où la pratique de la langue kurde est interdite, contrairement à l'Irak. Ainsi, les personnes qui ont activement contribué au développement de l'enseignement en langue kurde en Suède sont quasiment toutes originaires de Turquie. Toutes les maisons d'édition en langue kurde sont dirigées par des Kurdes de Turquie même si elles publient, en partie, des ouvrages en sorani21. Ces constats sont les signes d'un véritable besoin en matière linguistique et de (re)construction identitaire; besoin qui n'est pas exprimé (ou ne l'est peut-être pas de la même manière) chez les Kurdes d'Irak, qui, par ailleurs, disposent d'importants moyens au pays, notamment depuis 199122. Pour les Kurdes originaires de Turquie, jusqu'à une date très récente, l'Europe, et en particulier la Suède, était l'unique terre des possibles ; ce qu'elle n'est pas pour les Kurdes d'Irak. Ainsi, si l'utilisation de la langue kurde chez les Kurdes de Turquie est moins spontanée que chez les Kurdes d'Irak, la valeur politique qu'elle peut recouvrir est aussi, certainement, plus grande.

[17] La réussite de cet enseignement en direction des Kurdes de Turquie paraît mitigée. Au moment où les cours de kurde ont commencé à se mettre en place, beaucoup d'enfants kurdes se rendaient dans les classes de LCO en turC: les enfants des travailleurs kurdes mais aussi les enfants de responsables politiques (kurdes), ou même de professeurs de kurde, apprenaient le suédois et le turc et non le suédois et le kurde ${ }^{23}$. En 1985, Rexnegir met en évidence la non-utilisation par les Kurdes de leur droit à étudier leur langue maternelle, droit qu'ils revendiquaient pourtant, déjà en Turquie. L'auteur met en évidence la contradiction forte et douloureuse de ces personnes qui envoient leurs enfants dans les écoles qualifiées de 'kémalistes'24. Ainsi, en 1985, Rexnegir met l'accent sur l'important travail qu'il reste à faire et l'action que doit jouer la Fédération, mais aussi

\footnotetext{
21 Une exception à la règle : la maison d'édition - distribution Kitab-î Erzan est tenue par un Kurde d'Iran et travaille plus particulièrement en persan et en sorani. Par ailleurs, certains soranophones sont en contact avec les maisons d'édition kurmandjophones afin de les aider à la rédaction ou à la traduction de certains ouvrages.

${ }^{22} \mathrm{Au}$ Kurdistan d'Irak aujourd'hui, des livres, revues et journaux sont publiés légalement en grande quantité et la langue d'enseignement est le kurde. Toutefois, selon Dibistana Kurdî, le nombre de livres publiés en kurmandji en Suède entre 1936 et 1999 n'est que de peu supérieur à ceux publiés en sorani (respectivement 420 et 385).

${ }^{23}$ Rexnegir, Z. (1985) 'Li Swêdê Perwerde û Hindekariya Kurdî' [Éducation et enseignement de la langue kurde en Suède], Berbang 4, p. 4-6.

${ }^{24}$ Le matériel scolaire utilisé dans les classes de turc provient souvent directement de Turquie.
} 
Citation: Scalbert Yücel, Clémence (2006) 'La diaspora kurde en Suède. Conservation, production et diffusion d'un savoir linguistique', European Journal of Turkish Studies, Thematic Issue N5, Power, ideology, knowledge - deconstructing Kurdish Studies, URL : http://www.ejts.org/document771.html

To quote a passage, use paragraph (§)

toutes les associations et les publications kurdes en Suède : elles doivent soutenir ensemble la cause de la langue et de son enseignement, informer les parents, rendre plus performant l'enseignement et finalement le rendre exemplaire. Le nombre d'enfants apprenant le kurmandji en tant que LCO augmente progressivement. Toutefois, même si les enfants kurdes suivent des cours de kurde en Suède, d'après ce que nous avons pu observer, c'est assez rare que ces enfants (même s'ils ont une connaissance du kurde) parlent le kurde avec leur parents (dont l'un est parfois non kurde), leurs frères et sœurs ou leurs amis ${ }^{25}$. Cet enseignement permet néanmoins aux enfants de connaître leur langue - même s'ils ne la pratiquent pas - et de conserver leur kurdicité puisque la langue est conçue comme un vecteur de l'identité.

\section{Les programmes : langue et identité}

[18] Dès 1987, les enseignants de kurde confient à Ahmet Tîgrîs et à Ahmet Cantekîn (des éditions Haykurd) à Uppsala, à Haydar Diljen et à Bareş Battê à Stockholm, la tâche d'élaborer les programmes de langue kurde. Ces programmes trouvent leur illustration dans les différents manuels publiés en Suède pour les classes de LCO, soit directement par les maisons d'édition kurdes, soit par l'État suédois. La participation de l'État à la production de manuels se fait par l'intermédiaire de l'Agence pour l'immigration (Invandrarförlaget) dès 1979 et par celle de la Skolverket (Direction nationale des établissements scolaires ${ }^{26}$ qui soit finance la publication de ces ouvrages, soit les édite directement. La Skolverket édite également un dictionnaire suédois-kurde (réalisé par Reşo Zîlan, 2001), dans la collection Lexin. Cette collection est spécialement dévolue à la création de dictionnaires destinés au cours de $\mathrm{LCO}^{27}$.

\footnotetext{
${ }_{25}$ Ces observations sont confirmées par les entretiens réalisés par Ahmet Tîgrîs avec des d'élèves de classe de kurde. Si on parle kurde à la maison, on préfère parler suédois avec ses frères et ses amis. Citons l'élève Zinar : 'à la maison, on parle kurde. Mais parfois avec mes frères et sœurs, on parle en suédois. Le suédois, c'est facile...Quand il y a des mariages ou des fêtes, on se retrouve entre enfants kurdes et on parle en suédois. Parce que le suédois c'est facile' ('Em di mal de bi kurdî qise dikin. Lê carnan bi xwişk û birayên xwe re bi Swedî qise dikim. Swedî hêsa ye ... Dema dawet û şevên şayiyê çê dibin em zarokên Kurd tên bav hevdû û em bi Swedî qise dikin. Ji ber ku Swedî hêsa ye'). Berbang 68, 1990, p. 19.

${ }^{26}$ Créée en 1991, sous l'autorité du ministère de l'Éducation nationale et de la science, la Skolverket remplace la Skolöverstyrelsen (Direction Nationale de l'Enseignement Public).

${ }^{27}$ La décision de créer le projet LEX-IN (Lexikon för Invandrare - Dictionnaires pour étrangers) a été prise en 1979 par le Skolöverstyrelsen face à l'absence constatée de bons dictionnaires pour l'apprentissage des langues étrangères. Le projet est mené en collaboration par les organisations d'État pour l'immigration, pour les établissements scolaires ainsi qu'avec un nombre important d'universités. II a pour objectif de promouvoir
} 
Citation: Scalbert Yücel, Clémence (2006) 'La diaspora kurde en Suède. Conservation, production et diffusion d'un savoir linguistique', European Journal of Turkish Studies, Thematic Issue №5, Power, ideology, knowledge - deconstructing Kurdish Studies, URL : http://www.ejts.org/document771.html

To quote a passage, use paragraph (§)

[19] Les programmes des classes (1 à 9) de l'école obligatoire sont publiés dans le numéro 75 de Berbang en 1991. L'apprentissage de la lecture et de l'écriture se fait à partir de la classe 2. Dès la première classe, on commence à apprendre les danses, les contes et les chants kurdes (mais aussi suédois) et en classe 2, la culture kurde est évoquée. C'est à partir du niveau 2 (classes 4-5-6) que l'on commence à travailler sur la géographie du pays d'origine (étude de certains lieux du Kurdistan, repérage des 'voisins du Kurdistan', 'des pays occupants', des villes, fleuves et montagnes) ; l'histoire kurde mais aussi celle des fêtes nationales et en particulier de Newroz sont également enseignées. Dans le troisième niveau, toutes ces questions sont revues en détail et l'on découvre la littérature ${ }^{28}$. L'éducation dans cette langue-culture est donc aussi, dès ses débuts, une éducation à l'identité et même à l'identité nationale. Tous les attributs nationaux que mentionne, par exemple, Anne-Marie Thiesse (1999) sont effectivement présents dans ces programmes. Le but premier de ce matériel est de permettre l'apprentissage de la langue. Mais celle-ci étant pensée - et d'abord par les politiques migratoires suédoises - comme le support et le véhicule de la culture, les ouvrages doivent également traiter de cette culture et de cette identité : le dernier manuel publié, destiné aux classes de lycée, s'intitule d'ailleurs Le berceau de l'identité (Diljen, Erdem, Lewendi, Karim 2002) et se donne comme objectif principal le renforcement de l'identité kurde des élèves. La dernière phrase du livre s'adresse aux élèves en leur demandant ce que signifie pour eux le fait d'être kurde : les élèves, après avoir parcouru l'ensemble de l'ouvrage, devraient être capables de donner une réponse à cette question. Tous les élèves lient la kurdicité, qu'ils ont souvent ultérieurement appris à connaître et à aimer, à la langue : 'Mon identité, c'est la langue kurde' (Diljen, Erdem, Lewendi, Karim 2002 : 33). Ce lien très étroit entre langue et identité est fait en Turquie mais il est certainement renforcé en Suède où les activités linguistiques caractérisent une des principales activités de la diaspora.

[20] Dans les manuels publiés en Suède, les Kurdes sont présentés comme une nation d'immigrés et d'exilés. Les manuels s'ouvrent sur la mémoire du pays d'origine. Celui-ci est dépeint comme un paradis terrestre et sa nostalgie est clairement entretenue ${ }^{29}$.

le 'bilinguisme actif' chez les immigrés. 15 dictionnaires ont été publiés dans cette collection. Depuis janvier 2007, la Språkrådet (Conseil des Langues de Suède) est responsable du projet.

${ }^{28}$ Les programmes scolaires sont établis localement et varient selon les municipalités. Pour un exemple de programmes (ceux de la ville de Västerås), voir : http://modersmal.skolutveckling.se/nordkurdiska/plan/ 29 II en est de même pour ceux publiés ultérieurement en Turquie et utilisés dans les cours privés de langue kurde ouverts en 2004, mais très vite fermés par manque d'élèves. Voir Scalbert 2005. 
Citation: Scalbert Yücel, Clémence (2006) 'La diaspora kurde en Suède. Conservation, production et diffusion d'un savoir linguistique', European Journal of Turkish Studies, Thematic Issue №5, Power, ideology, knowledge - deconstructing Kurdish Studies, URL : http://www.ejts.org/document771.html

To quote a passage, use paragraph (§)

La diaspora se construit sans détour dans les classes de kurde. C'est ainsi que l'Europe est envisagée comme le lieu essentiel de préservation de l'identité contre l'assimilation mise en place par l'État turc, violemment dénoncée. Le professeur de kurde et les cours de kurde en Suède sont alors très vigoureusement célébrés. Les classes de kurde sont perçues comme des lieux de lutte contre l'assimilation menée par 'l'ennemi' turc ${ }^{30}$.

\section{L'école kurde en ligne : l'ubiquité de la langue}

[21] Le rôle de l'informatique est également important dans le domaine de l'éducation. L'exemple phare est celui de l'École kurde en Ligne, Dibîstana Kurdî, fondée en 2000 par un enseignant de kurde en Suède, Haydar Diljen, autour d'une équipe composée de Sabiha Otlu, Mahmud Lewendî, Kamal Khan ${ }^{31}$. Le premier but de ce site Internet ${ }^{32}$ est de dispenser en ligne des leçons de kurde : 'Son but premier est de devenir un lieu de dialogue direct entre les professeurs, les élèves et les familles kurdes. II peut être utilisé par les professeurs kurdes en classe avec leurs élèves, par les familles à la maison, par les enfants, seuls, sur leurs ordinateurs, ou bien par les associations kurdes avec leurs membres, enfants ou adultes'33. La Skolverket, séduite par l'école en ligne, l'a élu projet pilote, s'est engagée à le soutenir et l'a finalement accueilli sur son propre site Internet. La mairie de la ville de Västerås (chef-lieu du Comté de Västmanland, à l'Est du pays) est devenue propriétaire de ce projet désormais financé par l'État suédois. Ce projet pilote est le projet central du site Tema Modersmål (Thème Langue maternelle) de l'Agence suédoise pour le progrès de l'école (Myndigheten för skolutveckling), qui inclut 18 autres sites Internet préparés par les professeurs de LCO en Suède. Ce site a été récompensé par un prix de l'Union Européenne du fait de son travail pour le développement du multiculturalisme et du plurilinguisme. Avec cette école en ligne, la langue kurde et son enseignement ne sont plus cantonnés à un lieu précis (la Suède), ne sont plus entravés par les limites légales (la loi turque) et par les frontières internationales

\footnotetext{
30 Voir par exemple, Ferman, S. (1984) 'Beşa Kurdî Pîroz be' [Vive la section de kurde], Berbang 2, p. 3.

31 Tous ont travaillé comme professeurs de kurde en Suède. Haydar Diljen et Kamal Khan ont par ailleurs des compétences importantes en informatique. Seul Kamal Khan n'est pas originaire de Turquie.

$32 \mathrm{http://www.dibistanakurdi.com/}$

33 'Armanca wê ya sereke di navbera mamoste, şagird û malbatên kurd de bibe cihê dîalogeke rasterast. Mamosteyên kurdî digel şagirdên xwe dikarin di polan de, malbat li malê bi zarokên xwe re, zarok li ser kompûterê xwe bi xwe û komeleyên kurdan li komeleyan ligel endamên xwe yên biçuk û mezin bi kar bînin'. 'Dibistana Kurdî', http://www.dibistanakurdi.com/modules.php?name=Content\&pa=showpage\&pid=10. Consulté le 07.03.2007.
} 
Citation: Scalbert Yücel, Clémence (2006) 'La diaspora kurde en Suède. Conservation, production et diffusion d'un savoir linguistique', European Journal of Turkish Studies, Thematic Issue №5, Power, ideology, knowledge - deconstructing Kurdish Studies, URL : http://www.ejts.org/document771.html

To quote a passage, use paragraph (§)

(comme peut l'être la circulation de l'imprimé). L'école est accessible à tous, même depuis la Turquie.

\section{L'école et la norme}

[22] L'école permet de conserver la langue kurde, en usage ou en mémoire, pour les élèves scolarisés en Suède. Parce qu'elle est enseignée dans les classes et décrite dans les manuels, elle doit être normée. Certaines organisations kurdes en exil ou en diaspora travaillent à la normalisation de la langue, alors que rien n'a véritablement lieu en Turquie jusque dans les années 199034. C'est essentiellement le cas de l'Institut Kurde de Paris, fondé en 1983, avec sa revue de normalisation lexicale, Kurmancî, publiée à partir de 198735. Toutefois, la standardisation complète de la langue passe par la diffusion de cette norme ${ }^{36}$. La langue kurde (à destination des Kurdes de Turquie) n'a pas de vecteurs plus forts que la télévision et l'imprimé, sauf, peut-être, bien que pour un public limité, les écoles en Suède. Dès 1984, les professeurs kurdes en Suède prennent la décision d'utiliser l'alphabet latin de Hawar37. En 1987, Reşo Zîlan mentionne la préparation par certains professeurs d'un ouvrage rassemblant la terminologie qui doit être utilisée dans les différentes leçons ${ }^{38}$. Si, aujourd'hui, en Suède, les discussions relatives à la terminologie kurde sont encore en cours, certaines décisions sont prises, notamment au sein des écoles. C'est ce à quoi servent, entre autres, les réunions entre professeurs, organisées depuis $2002^{39}$. Ces normes seront ultérieurement transmises aux élèves. Les classes de kurde constituent donc une étape très importante dans la standardisation, car elles permettent, à l'échelle de la Suède, la diffusion d'une norme. Ces classes peuvent également jeter les bases, bien que numériquement limitées, de l'émergence d'un public lecteur en langue kurde. Elles peuvent éventuellement fournir un petit public à la création

\footnotetext{
34 En Turquie même, c'est l'Institut Kurde d'Istanbul qui se chargera en partie de cette tâche. Toutefois, les travaux des différentes associations kurdes de par le monde ne sont pas coordonnés.

35 Voir la revue Kurmancî http://www.institutkurde.org/publications/kurmanci/

${ }_{36}$ Nous utilisons ici la notion de standardisation dans le sens élaboré par Haugen (1966). Ce processus connaît quatre aspects : sélection de la norme, codification de la forme, élaboration de la fonction et acceptation par la communauté.

37 'Li Stockholmê di Xwendegeha Bilind a Mamostetiyê de Beşa Kurdî Vebû' [Une section de kurde a été ouverte à l'École Normale de Stockholm], Berbang 52, 1984, p. 12-13. Sur le rôle de la revue Hawar dans la standardisation du kurmandji, voir Scalbert 2006.

38 'Mamosteyên Kurd Dîplome girtin' [Les professeurs kurdes ont reçu leurs diplômes], Berbang, mars 1987, pp. 8-9.

39 Toutefois, il existe, depuis le 4 avril 1987, une union des professeurs kurdes en Suède qui se réunit pour traiter des questions relatives à l'enseignement. Voir Roja Nû 17, 1987, p. 31.
} 
Citation: Scalbert Yücel, Clémence (2006) 'La diaspora kurde en Suède. Conservation, production et diffusion d'un savoir linguistique', European Journal of Turkish Studies, Thematic Issue №5, Power, ideology, knowledge - deconstructing Kurdish Studies, URL : http://www.ejts.org/document771.html

To quote a passage, use paragraph (§)

littéraire qui se développe de manière spectaculaire en Suède, elle aussi largement favorisée par le soutien étatique à la production culturelle.

\section{La centralité de la Suède dans le développement des activités littéraires kurdes}

\section{L'édition}

\section{L'aide publique à l'édition}

[23] Une politique culturelle d'aide aux activités artistiques se met en place en Suède dès les années 1930. Elle est restructurée en 1974 avec une résolution du parlement qui définit les objectifs de la politique culturelle de l'État ${ }^{40}$ : c'est dans ce contexte de reformulation de la politique culturelle nationale qu'est créé le Conseil national suédois pour les affaires culturelles (Kulturrådet). Le Conseil poursuit les objectifs de la politique culturelle étatique. Outre l'information, la coopération, sa plus grande tâche est l'attribution de bourses dans les domaines de la danse, du théâtre, de la musique, de la littérature, des bibliothèques, des revues artistiques, de la muséologie, des expositions, et des arts visuels ${ }^{41}$. Seules les institutions et les organisations peuvent recevoir ces bourses.

[24] Les maisons d'édition ou les revues kurdes, en tant qu'organisations ou associations, peuvent bénéficier de ces aides. En ce qui concerne les maisons d'édition, les aides sont versées, non pas pour la maison en général, mais pour la publication de tel ou tel ouvrage, jugé particulièrement intéressant: la maison envoie le livre édité, ou la maquette du livre et un comité de la Kulturrådet décidera ou non de le subventionner. La majorité des maisons d'édition kurdes ont perçu une aide financière au moins une fois (Tayfun $1998: 30$ ).

\footnotetext{
40 Dans le contexte du Programme du Conseil de l'Europe pour 'l'évaluation des politiques culturelles nationales', la politique culturelle a été repensée et, en 1996, ses objectifs sont sensiblement redéfinis : sauvegarder la liberté d'expression et créer des opportunités pour en user; travailler à la création d'opportunités pour tous de participer à la vie culturelle et d'engager des activités créatives propres ; promouvoir le pluralisme, le renouveau artistique et culturel et la qualité en contrant les effets négatifs du commercialisme ; faire en sorte que la culture puisse devenir une force dynamique indépendante ; préserver et utiliser l'héritage culturel ; promouvoir l'éducation culturelle; promouvoir les échanges culturels internationaux. Voir $\quad$ http://www.kulturradet.se/index.php?realm=357 http://www.kulturradet.se/ovfiles/CulturalObjectives.pdf. Consulté le 07.03.2007.

${ }^{41}$ http://www.kulturradet.se/index.php?realm=383. Consulté le 07.05.2007.
} 
Citation: Scalbert Yücel, Clémence (2006) 'La diaspora kurde en Suède. Conservation, production et diffusion d'un savoir linguistique', European Journal of Turkish Studies, Thematic Issue №5, Power, ideology, knowledge - deconstructing Kurdish Studies, URL : http://www.ejts.org/document771.html

To quote a passage, use paragraph (§)

\section{Aperçu des maisons d'édition kurdes}

[25] Les maisons d'édition kurdes font une apparition massive au début des années 1980 en Suède. Avant cette date, elles sont rares et un certain nombre d'auteurs kurdes publient dans les maisons étatiques suédoises. Le fait que ces maisons puissent bénéficier d'aides financières de la part du gouvernement suédois explique qu'elles apparaissent principalement dans ce pays et non pas ailleurs - l'Allemagne, par exemple, ne connaît pas une telle activité éditoriale alors que la population kurde y est bien plus importante. Ceci explique aussi la durée de vie relativement longue de ces maisons ${ }^{42}$.

[26] Nous avons répertorié vingt-neuf maisons d'édition kurdes en Suède - toutes fondées et dirigées par des personnes originaires de Turquie à une exception près. Les premières maisons importantes apparaissent en $1980^{43}$ : Deng ('La Voix', dernière publication en 1998), Roja Nû ('Le Nouveau jour')44, Çanda Kurdî ('Culture kurde', 1981 1992), Jîna Nû ('La Vie nouvelle', 1984-1999), Haykurd (1987-1994) et Welat ('Le Pays', 1988-1994). D'autres maisons sont ouvertes à la fin des années 1980 et fonctionnent encore aujourd'hui : Apec, Pencinar, Çanda Nûjen ('La Culture moderne', qui devient Helwest en 1997) et enfin Nûdem ('La Nouvelle ère', 1992). Après Nûdem, aucune 'grande' maison n'est fondée. Les maisons créées après 1992, telles Pelda (1993) ou Jîndan (1996), sont de taille réduite (moins de 10 titres) et ne servent à publier quasiment que les œuvres de leur propriétaire, respectivement Laleş Qaso et Maruf Yilmaz. On peut également mentionner les éditions kurdes qui publient essentiellement en turc et qui n'ont que quelques titres en kurde : Mezopotamya, et Rewşen ('La lumière') ont publié respectivement deux et cinq titres. Mentionnons enfin la seule maison féminine, les éditions de l'association des femmes kurdes en Suède (Weşanên Komeleya Jinên Kurd li Swêdê, 1989-1992 ; cinq titres).

[27] Les maisons que nous qualifions ici d'importantes le sont d'une part par leur nombre de titres (plus de vingt voire, pour les plus grosses, plus de cinquante), d'autre part

\footnotetext{
42 En effet, les maisons d'édition ou les publications périodiques qui verront le jour en Turquie dès 1991 ont des durée de vie bien plus courte, généralement à cause de problèmes économiques. Les procès engagés par l'Etat contre ces maisons et les lourdes amendes qu'elles encourent renforcent encore l'instabilité économique.

43 Les premières maisons d'édition kurdes sont créées dans les années 1970. Bahoz est créé vers 1972, par des Kurdes de Turquie proches du Parti Démocrate du Kurdistan - Irak. Pale, proche des Partisans de la libération nationale du Kurdistan (Kurdistan Ulusal Kurtuluşcuları), publie une revue de 90 pages en turc et en kurde. À elles deux, elles publient uniquement trois livres en kurde (Cigerxwîn et Nureddin Zaza).

${ }^{44}$ Qui est toujours ouverte, même si elle n'a pas publié depuis 1999.
} 
Citation: Scalbert Yücel, Clémence (2006) 'La diaspora kurde en Suède. Conservation, production et diffusion d'un savoir linguistique', European Journal of Turkish Studies, Thematic Issue №5, Power, ideology, knowledge - deconstructing Kurdish Studies, URL : http://www.ejts.org/document771.html

To quote a passage, use paragraph (§)

pour leur rayonnement. Welat, par exemple, a peu de titres mais a publié Mehmet Uzun et Hesenê Metê, deux des écrivains kurdes contemporains de Turquie les plus reconnus aujourd'hui. Deng s'est illustré dans la (re)publication de documents d'archives ou de la littérature orale, Haykurd dans celle de manuels pour l'apprentissage de la langue, en particulier à destination des enfants. Ces maisons ne sont pas qualifiées de grandes maisons par la taille de l'entreprise ou le nombre d'employés. En effet, le travail d'édition est généralement celui d'un seul homme : le propriétaire de la maison. Seules les éditions Apec présentent un caractère d'entreprise avec des employés mais ceci n'est rendu possible que par la multifonctionnalité de la maison : c'est aussi une imprimerie et une agence de graphisme.

[28] Ces maisons sont souvent liées au monde politique, au moins à l'origine, même si les ouvrages politiques sont très peu nombreux. C'est souvent sur des bases d'affinités idéologiques que se retrouvent les personnes gravitant dans ces maisons. II en est de même pour les publications périodiques, présentes en Suède depuis les années 1960, qui, très politisées, sont souvent rattachées à un parti45. Revues politiques, elles étaient, par ailleurs, essentiellement publiées en turc. C'est pour se défaire de ces liens d'avec le politique que Firat Cewerî lance la revue Nûdem, revue littéraire et artistique, puis les éditions du même nom avec cette idée centrale : l'indépendance. Cewerî souhaite casser ainsi une 'tradition', celle du poids du politique dans le domaine éditorial, et ouvrir une voie nouvelle en éditant exclusivement en kurde ${ }^{46}$.

[29] Ainsi, l'édition en langue kurde se développe alors en Suède dès les années 1980 alors qu'elle est, du fait de l'interdiction de la langue kurde, encore inexistante en Turquie (graphique 1$)^{47}$. Les aides financières permettent une activité relativement longue mais aussi une indépendance vis-à-vis des partis politiques kurdes, pour ceux qui le souhaitent. Les éditeurs toutefois ne vivent généralement pas de leur activité éditoriale.

\footnotetext{
45 Les publications périodiques, comme les maisons d'édition peuvent aussi percevoir des aides financières de l'État. Nûdem, revue indépendante en touche comme les revues politiques. Voir Tayfun $1998: 34$. 46 Voir : Cewerî, Firat (1992) 'Çend Gotin' [Quelques mots], Nûdem 1, p. 3 ; Cewerî, F. (2002) 'Çîroka min û Nûdemê' [Mon histoire et celle de Nûdem], Nûdem 40, pp. 4-28. Notons que beaucoup de revues kurdes sont bilingues (turc-kurde).

${ }^{47}$ Nous avons recensé 459 ouvrages publiés en kurmanci en Suède jusqu'en 2005 (Scalbert-Yücel 2005). Alakom en mentionne 420 (et 375 en sorani, 23 en zazaki) (2006 : 197).
} 
Citation: Scalbert Yücel, Clémence (2006) 'La diaspora kurde en Suède. Conservation, production et diffusion d'un savoir linguistique', European Journal of Turkish Studies, Thematic Issue №5, Power, ideology, knowledge - deconstructing Kurdish Studies, URL : http://www.ejts.org/document771.html

To quote a passage, use paragraph (§)

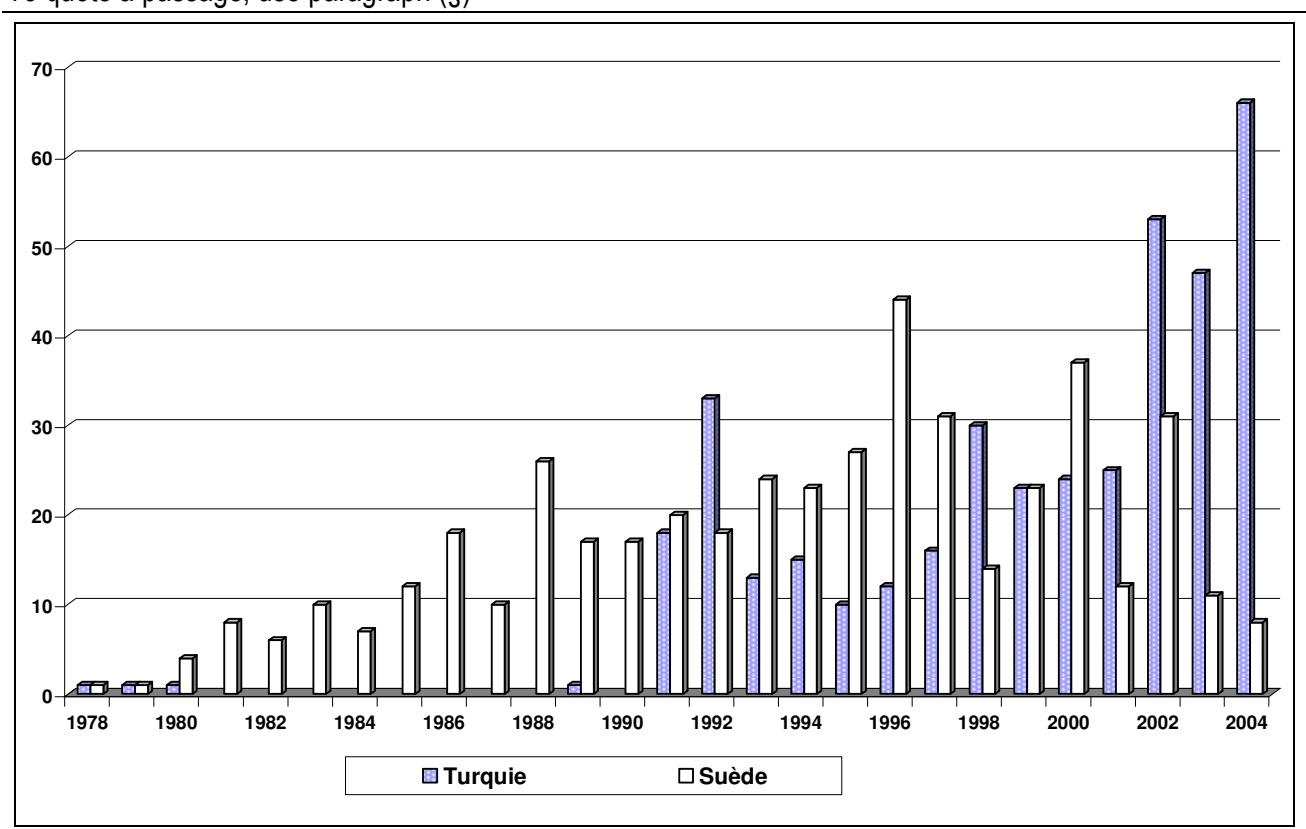

Graphique 1: Nombre de titres publiés en kurmandji en Suède et en Turquie (1978-2004)

Source : Scalbert Yücel 2005 (Pour la production en Suède durant les années 2003-2004, nous avons uniquement consulté le site Internet de la bibliothèque nationale suédoise. Nous présentons ces années uniquement à titre indicatif).

\section{Le livre pour enfant - le meilleur marché}

[31] L'enseignement en LCO qui prend son essor au début des années 1980 devrait permettre, théoriquement et idéalement, l'émergence d'un lectorat. Inversement, la publication devrait aussi créer un public. L'enseignement du kurde dans les écoles suédoises nécessite un certain nombre d'ouvrages scolaires et d'outils pédagogiques d'où, certainement, l'intérêt de ces publications pour les maisons d'édition. L'État suédois apporte également une aide au développement du livre pour enfants et du livre scolaire ${ }^{48}$. Les maisons d'édition gouvernementales ont édité quelques livres. II s'agit en général des organismes du gouvernement liés soit à l'éducation, avec l'Institut national pour le matériel éducatif (Statens Institut för Läromedel - SIL, dépendant de la Skolverket), soit à l'immigration, avec l'Agence suédoise pour l'immigration (Invandrarförlaget - Statens InvandrarVerk). Ainsi, jusqu'en 1990, SIL aurait publié huit livres en kurde, livres qui traitent tous de la langue et de son apprentissage ${ }^{49}$. Dès la fin des années 1970 et encore

\footnotetext{
${ }^{48}$ Cet investissement de l'Etat suédois dans l'édition de matériel pédagogique et de livres pour enfants est certainement lié à l'enseignement des LCO ; on peut peut-être émettre l'hypothèse qu'il suit la parution de rapports réalisés en 1977 et 1982 par Myklos Gulyas mettant en évidence l'absence de livre pour enfants, notamment pour les enfants kurdes. Ces rapports sont mentionnés par Alakom 2006 : 180-181.

49 Zîlan, Reşo (1990) 'Pêşdeçûna Ziman û Rewşa Weşanên Kurdî li Ewropayê' [Le progrès de la langue et la situation des éditions en kurde en Europe], Berbang 65, p. 18-21.
} 
Citation: Scalbert Yücel, Clémence (2006) 'La diaspora kurde en Suède. Conservation, production et diffusion d'un savoir linguistique', European Journal of Turkish Studies, Thematic Issue №5, Power, ideology, knowledge - deconstructing Kurdish Studies, URL : http://www.ejts.org/document771.html

To quote a passage, use paragraph (§)

au début des années 1980, le Bureau suédois pour l'immigration publie une petite dizaine de livres kurdes (livre pour enfants et livres de contes).

[32] De nombreuses maisons d'édition consacrent une part importante à l'édition du livre pour enfants qui constitue près d'un quart de la production éditoriale kurde en Suède ${ }^{50}$. Même si nous ne disposons pas de sources relatives aux différents livres qui ont été subventionnés, il semble assez clair que les livres pour enfants sont largement financés par l'État ${ }^{51}$. Les premières maisons d'édition se tournent vers le livre pour enfants, et en particulier Çanda Kurdî52. Lorsque Ahmet Cantekîn fonde les éditions Haykurd, son but principal est de publier des livres pour enfants (dix-huit titres sur vingt). Contrairement aux autres maisons, il ne publie que sa propre production (et non des traductions). Après ce travail initié par Çanda Kurdî et Haykurd, les autres maisons d'édition s'intéressent à la publication du livre pour enfants, qui sont généralement des traductions. L'édition littéraire se développe également avec l'émergence d'une écriture littéraire : elle représente plus d'un tiers de la production éditoriale.

\section{L'écriture littéraire}

[33] L'État suédois ne propose pas uniquement des aides à l'édition, mais également des aides à l'écriture. Ces aides proviennent essentiellement du Fonds suédois des auteurs (Sveriges Författarfond - SVFF). En 2007, le Fonds est administré par un bureau constitué de vingt huit membres. Le gouvernement nomme le président et sept membres, l'Union suédoise des Ecrivains (Sveriges Författarförbund) nomme seize membres, l'Association suédoise des Illustrateurs (Svenska Tecknare) et l'Association suédoise des Photographes (Svenska Fotografers Förbund) appointent chacune deux membres. Les aides financières attribuées sont de différents types. La principale provient des emprunts en bibliothèque sur lesquels les auteurs perçoivent un pourcentage : une compensation est payée pour les emprunts de livres écrits en suédois, traduits en suédois, ou écrits par des auteurs résidant de manière permanente en Suède. Tous les auteurs

50 Données issues de l'analyse de la bibliographie des ouvrages publiés en kurmancî en Suède. Voir Scalbert Yücel 2005.

51 Selon Ali Çiftçi, la maison publie environ quinze livres par an, seulement deux ou trois d'entre eux reçoivent une aide. En général, ce sont les livres pour enfants (traduits du suédois) qui sont subventionnés. Entretien avec Ali Çiftçi, Stokholm, 2 octobre 2003. Voir aussi Tayfûn 1998.

52 Cette maison publie vingt-six livres pour enfants sur un total de vingt-neuf livres. Sur les vingt-six livres pour enfants qu'elle publie, vingt-cinq sont des traductions. 
Citation: Scalbert Yücel, Clémence (2006) 'La diaspora kurde en Suède. Conservation, production et diffusion d'un savoir linguistique', European Journal of Turkish Studies, Thematic Issue №5, Power, ideology, knowledge - deconstructing Kurdish Studies, URL : http://www.ejts.org/document771.html

To quote a passage, use paragraph (§)

cependant n'en profitent pas et nous supposons que la plupart des auteurs kurdes n'en bénéficient pas, cette aide n'étant pas mentionnée dans nos sources ${ }^{53}$. Cette aide spécifique correspond à $35 \%$ du budget 2004 du Fonds et il est estimé qu'elle correspondra à $37 \%$ du budget en 2007. Outre cette 'rémunération basée sur les emprunts', il existe une 'rémunération garantie' qui est supérieure à la somme maximale que le Fonds peut débourser en regard des emprunts ; elle est versée à des personnes qui veulent exercer les métiers d'auteur, de traducteur ou d'illustrateur comme activité principale. Le caractère de leur production est également pris en considération. Cette rémunération est versée en 2007 à 191 auteurs, traducteurs et illustrateurs et correspond à $22 \%$ du budget. L'argent restant est appelé la 'partie libre du Fonds'. C'est en quelque sorte, un 'fonds de solidarité' dans l'intérêt des auteurs, traducteurs et illustrateurs. Cette partie libre est utilisée pour le versement de bourses (stipendium) et d'aides (bidrag) diverses aux auteurs dont les travaux se trouvent dans les bibliothèques suédoises. Le bureau examine les candidatures en tenant compte des besoins de l'auteur et des qualités de son travail. II s'agit de bourses de travail de 5 ans (90 000 à 99000 couronnes $^{54}$ par an, imposables), de un à deux ans (40000 - 80000 couronnes par an, non imposables) ou de bourses de voyage. Des aides sous forme de retraites peuvent être versées à des auteurs qui ont déjà une petite retraite nationale et dont la production est qualifiée de satisfaisante (d'un point de vue qualitatif et quantitatif). Des aides sont également attribuées pour des auteurs en crise financière, pour des activités littéraires particulières (auteurs travaillant dans le domaine artistique ou pour des échanges culturels internationaux) ${ }^{55}$.

[34] Comme d'autres auteurs d'origine immigrée et résidant en Suède, une partie des auteurs kurdes bénéficient, au moins depuis 1984, et encore aujourd'hui, de ces aides issues de la partie libre du Fonds ${ }^{56}$. II n'y a apparemment pas de comité spécial pour les langues autres que le Suédois. Le Fonds engage des lecteurs qu'il connaît, ou sur recommandation, et essaye d'en changer au maximum. Ceux-ci ne prennent pas de décision mais sont là pour informer le Fonds sur la qualité des travaux de l'auteur ${ }^{57}$. Ces ressources attribuées par le Fonds permettent à l'auteur de se consacrer à son travail, de

\footnotetext{
53 La rémunération dépend du nombre d'emprunts. Si la rémunération est inférieure à 1420 couronnes (qui correspondent à 2000 emprunts), l'auteur ne sera pas payé.

54 Une Couronne suédoise équivaut en 2007 à 10 centimes d'Euro.

${ }^{55} \mathrm{http://www.svff.se/fondeng.htm} \mathrm{Consulté} \mathrm{le} \mathrm{25.06.2007.}$

56 Tayfûn 1998: 25.

57 Information communiquée par mail par le Fonds Suédois des Auteurs.
} 
Citation: Scalbert Yücel, Clémence (2006) 'La diaspora kurde en Suède. Conservation, production et diffusion d'un savoir linguistique', European Journal of Turkish Studies, Thematic Issue N5, Power, ideology, knowledge - deconstructing Kurdish Studies, URL : http://www.ejts.org/document771.html

To quote a passage, use paragraph (§)

pouvoir faire ses recherches, sans être forcément contraint à mener une activité professionnelle en parallèle.

[35] L'impossibilité d'écrire en kurde en Turquie même jusqu'au début des années 1990 explique l'émergence de ce pôle suédois. Toutefois, l'existence de ce Fonds est pour beaucoup dans le développement d'un pôle littéraire en Suède particulièrement et dans l'émergence de l'écrivain kurde dans ce pays même. Selon Rohat Alakom, 70 \% des écrivains kurdes vivant en exil résideraient en Suède (Alakom 2006 : 195). Les écrivains kurdes aujourd'hui les plus reconnus vivent tous en Suède.

\section{La mémoire papier}

[36] Le rôle de conservatrice que joue la diaspora s'exprime dans ses travaux d'archivage. Le premier consiste à mettre par écrit les traditions orales qui, du fait $d u$ processus de 'turcisation' et 'd'assimilation' en Turquie, pourraient être oubliées. L'archivage ainsi est d'abord celui qui concerne la collecte et la conservation (grâce à la publication) des traditions orales. Cette forme de mise en mémoire, destinée à l'alimentation de la mémoire collective du groupe, se rencontre partout, en exil d'abord, puis en Turquie par la suite. Mais, il a pris une ampleur considérable en Suède où, en particulier, deux maisons s'illustrent dans le passage à l'écrit et dans la conservation, des traditions orales : Deng et Pencinar ne publient quasiment exclusivement que des contes, proverbes ou épopées.

[37] L'archivage concerne aussi tous types de documents publiés. En Turquie, jusque dans les années 1990, certains éléments de bibliothèques privées, livres, revues ou brochures, finissaient dans le poêle en hiver, enfouis dans les jardins où ils étaient rongés par l'humidité en été. Rares sont les personnes qui, aujourd'hui, possèdent chez elles, en Turquie de grandes archives. Aucune bibliothèque ne demeurait intacte après le passage de la police ${ }^{58}$. Les archives des associations, organismes ou partis politiques kurdes en Turquie étaient les plus vulnérables et les plus éphémères. L'archivage fut d'abord le fait de certaines personnes privées en Europe, puis celui d'organisations ou d'organismes. La première ouverte est certainement la bibliothèque de I'Institut Kurde de Paris mais

${ }^{58}$ Le film Viziontele Tuuba (YıImaz Erdoğan, Turquie, 2004) qui traite, de manière humoristique, du coup d'État de 1980 montre une scène caractéristique : la bibliothèque de la petite ville d'Hakkari, si difficilement ouverte et achalandée est la première visée et dévastée dans la nuit du 11 au 12 septembre 1980. 
Citation: Scalbert Yücel, Clémence (2006) 'La diaspora kurde en Suède. Conservation, production et diffusion d'un savoir linguistique', European Journal of Turkish Studies, Thematic Issue №5, Power, ideology, knowledge - deconstructing Kurdish Studies, URL : http://www.ejts.org/document771.html

To quote a passage, use paragraph (§)

dépendante de l'organisme. C'est en 1997 qu'ouvre, comme institution indépendante ${ }^{59}$, la Bibliothèque kurde (Kitêbxaneya Kurdî) à Stockholm. Elle est établie en février 1996 avec le support financier de la Fondation de la culture du futur (Stiftelsen framtidens kultur), du gouvernement suédois et de la ville de Stockholm ${ }^{60}$. Le projet fut monté à l'initiative de Nedim Dağdeviren et de son Kurdish Publishing Centre (fondé en 1995). La bibliothèque reçoit également, pour un projet d'archivage, des aides de l'agence gouvernementale SIDA (Swedish International Development Cooperation Agency) ${ }^{61}$. Les objectifs de la bibliothèque sont relativement simples et brièvement présentés sur le site Internet: collecter, préserver, diffuser et mettre à disposition de chacun la littérature kurde ${ }^{62}$. La collection de la bibliothèque est composée de tout matériel imprimé traitant des Kurdes et du Kurdistan : livres, journaux et revues, brochures, mais aussi photographies, matériels vidéo et audio.

[38] Un projet de conservation et d'archivage est également mené par Goran Cano, responsable de Sara Distribution (Stockholm). Sara a été fondée en 1987 comme maison d'édition et de distribution. Elle se définit aujourd'hui comme le centre pour la protection et la diffusion de la langue, de la culture et de la littérature kurde (Navenda Parastin û Belavkirina Ziman, Wêje û Çanda Kurdî). Sara n'a publié que quelques livres en langue kurde et s'est essentiellement tournée vers la collecte et la distribution. En tant que distributeur, elle possède également une boutique de livres, riche de 20000 titres (en kurde ou sur les Kurdes ${ }^{63}$. Sara Distribution collecte tous les livres sur les Kurdes ou en kurde. Elle travaille en collaboration avec les maisons d'édition kurdes en Suède, en Irak et en Iran - elle n'a pas de contact en Turquie. Après avoir centralisé les livres, Sara les diffuse dans le monde entier. Les individus ont la possibilité de s'achalander en livres chez Sara, seule agence à rassembler l'essentiel des livres kurdes. Mais la plus grande fierté de Goran Cano est la constitution de sections kurdes dans de grandes bibliothèques. II est en

\footnotetext{
59 Elle deviendra 'fondation' en 1999.

${ }^{60}$ Certains projets internes à la bibliothèque sont subventionnés par la Ville de Stockholm, the General Library Association of Sweden, et l'Institut suédois.

Voir http://www.kurdishlibrary.org/Kurdish_Library/EnglishKL/Presentation_Eng.htm. Consulté le 07.03.2007.

Sur cette bibliothèque et les aides qu'elle a reçues, voir Tayfûn $1998: 55$.

61 Voir http://www.sida.se/shared/isp/download.jsp?f=SIDA1635en+Literature.pdf\&a=2441 Consulté le 07.03.2007. Cette agence gouvernementale, liée au ministère des Affaires étrangères, est responsable de la plupart des contributions suédoises dans le domaine de coopération internationale pour le développement. Elle a financé plusieurs projets dans la diaspora kurde (Tayfûn $1998: 35$ ).

62 http://www.kurdishlibrary.org/Kurdish_Library/EnglishKL/Presentation_Eng.htm Consulté le 07.03.2007.

63 http://www.saradistribution.com/ Consulté le 07.03.2007.
} 
Citation: Scalbert Yücel, Clémence (2006) 'La diaspora kurde en Suède. Conservation, production et diffusion d'un savoir linguistique', European Journal of Turkish Studies, Thematic Issue №5, Power, ideology, knowledge - deconstructing Kurdish Studies, URL : http://www.ejts.org/document771.html

To quote a passage, use paragraph (§)

effet le fournisseur de la bibliothèque de l'Université de Californie (Berkeley), de la British Library, de la New York Public Library, de la Library of Congress et de la Stockholm Internationella Bibliotek ${ }^{64}$.

\section{La diaspora et le pays d'origine, des relations équivoques}

[39] La diaspora kurde en Suède, 'sanctuaire', occupe ainsi de manière très claire les fonctions de conservatoire de la langue et de l'identité - mémoire qui y est liée. Elle joue aussi un rôle créatif par l'intermédiaire des éditeurs et des auteurs. Les auteurs notamment jouent véritablement ce rôle quand, par exemple, ils introduisent dans la littérature de langue kurmandji des genres tels que le roman, alors totalement inexistant chez les Kurdes de Turquie. Outre son rôle de créatrice, outre le fait qu'elle préserve la mémoire de l'origine, la diaspora diffuse aussi cette mémoire et ses créations parmi la nouvelle génération: celles des enfants kurdes scolarisés en Suède. Cette diaspora fonctionne un temps en cercle quasiment fermé : si des réseaux se développent au sein des différents pays européens, la diaspora n'a quasiment pas de contact avec la Turquie. La situation change à partir du début des années 1990 et la diaspora va développer ses activités en direction du pays d'origine. Ceci correspond à la période de libéralisation vis-àvis de la question de la langue et de l'expression d'une spécificité kurde: un premier assouplissement de la législation turque a lieu, en 1991, sous la présidence de Türgüt Özal65 ; il est suivi, en 2002, par l'autorisation de l'utilisation de langues autres que le turc dans les émissions de télévisions et de radios et leur enseignement dans des cours privés (loi $n^{\circ} 4771$ du 3 août 2002). Quelques exemples serviront à illustrer le rôle crucial que jouent les relations progressivement nouées entre les organisations kurdes en diaspora et en Turquie dans le développement des activités de la diaspora en Turquie. Pourtant, des tensions sont également visibles entre la diaspora et l'origine qui pourraient, à terme, amener à redéfinir le rôle de cette première.

\footnotetext{
${ }^{64}$ Azadiya Welat, 6-12 juillet 2002. http://www.saradistribution.com/AzadiyaWelat.htm

${ }^{65}$ La loi $n^{\circ} 2932$ de 1983 relative aux publications en d'autres langues que le turc est modifiée et permet alors l'usage des 'langues et dialectes locaux' dans les enregistrements audiovisuels et dans les autres moyens de communication.
} 
Citation: Scalbert Yücel, Clémence (2006) 'La diaspora kurde en Suède. Conservation, production et diffusion d'un savoir linguistique', European Journal of Turkish Studies, Thematic Issue №5, Power, ideology, knowledge - deconstructing Kurdish Studies, URL : http://www.ejts.org/document771.html

To quote a passage, use paragraph (§)

[40] La diaspora joue d'abord un rôle dans le domaine de la scolarisation et de la formation en direction des Kurdes résidant en Turquie. Un exemple marquant est celui de la formation de 'spécialistes de la langue kurde' résidant en Turquie. Ce projet est lancé dans la seconde moitié des années 1990 et est permis grâce à la collaboration entre la Fondation kurde d'Istanbul (Kürt-Kav) et la Fondation kurde de Stockholm. Chapeauté par cette dernière, le projet est financé par elle mais aussi par de riches Kurdes66 ou encore par le Parti social démocrate ou le Fonds Olof Palme. L'Université d'Uppsala, dans laquelle sont formés ces futurs spécialistes, ne rémunère pas les professeurs et ne fait finalement que prêter son nom pour délivrer les diplômes. La Fondation Kürt-Kav se charge du recrutement des candidats en Turquie. La préférence est donnée à des écrivains, éditeurs, professeurs ou journalistes qui pourront mettre à profit leur formation dès leur retour au pays. Trois promotions (environ trente personnes au total) ont été ainsi formées depuis 1996. Le principe est un stage de six mois pour ces personnes qui rentrent en Turquie diplômées, prêtes à assurer une activité d'enseignement ou de traduction - interprétariat. Un autre exemple des plus significatif dans le domaine de l'éducation est un projet de l'agence gouvernementale SIDA relatif à la publication et à la diffusion de livres pour enfants et qui associe la Fondation kurde de Stockholm à l'Institut Kurde d'Istanbul en 2004. La coordination entre les deux organismes passe par le Consul suédois à Istanbul. La Fondation a préparé, avec des auteurs résidant en Suède, une vingtaine de livres. Ils ont été imprimés en Turquie par l'Institut Kurde d'Istanbul, puis diffusés gratuitement par la mairie de Diyarbakir lors d'un festival en mai 200467. Du fait de la situation en Turquie, où l'enseignement en langue kurde est encore extrêmement limité et non institutionnalisé, la Suède conserve son rôle dominant dans ce domaine ${ }^{68}$. En revanche, dans le domaine littéraire et éditorial, si le rôle de la diaspora a été essentiel en Turquie même dans les années 1990, il semble être en passe de décliner aujourd'hui.

\footnotetext{
66 Un appel de la Fondation culturelle kurde à Stockholm est lancé dans Berbang $\left(n^{\circ} 117,2000\right)$ afin que chacun, selon ses moyens, fasse une donation qui servirait à financer les projets en cours : cours de langue à Uppsala, préparation d'un CD-Rom d'enseignement du kurde.

67 http://www.enstituyakurdi.org/modules.php?name=News\&file=article\&sid=78. Consulté le 07.03.2007.

68 En effet, des campagnes importantes relatives à l'enseignement en langue kurde ont été lancées en Turquie à partir de 2001, notamment dans les universités. Après les réformes de 2002, il a été autorisé d'enseigner le kurde dans des établissements privés. Les premiers cours débutent à Batman (22 janvier 2004), à Urfa (13 mars 2004), et à Adana. Puis ils sont ouverts à Van (5 avril 2004), à Diyarbakir (14 septembre 2004), à Istanbul (septembre 2004) et à Kızıltepe (16 novembre 2004). L'Institut kurde d'Istanbul les chapeaute tous, prépare et édite les manuels. Toutefois, ces cours sont fermés très vite, faute d'élèves, de moyens ou de motivation. Aujourd'hui, aucun d'entre eux n'est en fonctionnement.
} 
Citation: Scalbert Yücel, Clémence (2006) 'La diaspora kurde en Suède. Conservation, production et diffusion d'un savoir linguistique', European Journal of Turkish Studies, Thematic Issue №5, Power, ideology, knowledge - deconstructing Kurdish Studies, URL : http://www.ejts.org/document771.html

To quote a passage, use paragraph (§)

[41] Dans les années 1980, une littérature contemporaine de langue kurmandji se développe en Suède. Durant ces années, aucun auteur n'écrit en kurmandji en Turquie. Ainsi, lorsque les premières maisons d'édition kurdes ouvrent leurs portes en Turquie à partir de 1991, les livres publiés sont pour beaucoup ceux d'auteurs 'classiques', souvent décédés, des rééditions des quelques ouvrages publiés en Turquie dans les années 1970 ou des rééditions d'ouvrages publiés en Suède ${ }^{69}$. Si les auteurs kurdes en Suède sont reconnus par un petit cercle en Suède et en Europe, parfois traduits ou aidés par l'État, ils restent encore, pour la plupart, très éloignés et inconnus de leur lectorat potentiel. En 1996, Firat Cewerî, propriétaire des éditions Nûdem écrivait de manière extrêmement significative : 'Hors du pays, des maisons d'édition kurdes ont été créées en très grand nombre, des organisations démocratiques, des organisations culturelles ont été créées, des journaux et des revues en kurde ont été publiés, des films en kurde ont été réalisés. On peut dire qu'une renaissance de la langue et de la littérature kurde avait commencé. Mais, malheureusement, ce mouvement culturel kurde est resté cantonné à l'extérieur du pays, il n'a pas atteint nos compatriotes au pays. [...] Le travail culturel qui devint brillant en Europe en général et en Suède en particulier est resté replié sur lui-même'70. Tous les auteurs cherchent alors progressivement à sortir de l'éloignement géographique mais aussi littéraire, et à se faire connaître et reconnaître auprès d'un plus large public en Turquie. Dès les premiers moments de la libéralisation et avec l'ouverture des premières maisons d'édition kurde en Turquie (1991), les auteurs résidant en Suède affichent leur volonté de s'y faire publier : Mehmet Uzun y est réédité dès 1992, Firat Cewerî y est édité en 1993. C'est une opportunité pour les auteurs qui peuvent ainsi se faire connaître en Turquie. C'est aussi une opportunité pour les nouvelles maisons d'édition qui n'ont encore

${ }^{69}$ Ceci est apparaît extrêmement clairement lorsque l'on analyse l'ensemble des ouvrages publiés en 1991 : une réédition d'un ouvrage de Musa Anter, membre de l'Institut Kurde d'Istanbul, publié en Turquie en 1965 ; une réédition d'un ouvrage de Baran publié auparavant en Suède, deux ouvrages de Bedir Khan, dont une réédition d'un livre publié en Suède, une réédition d'un dictionnaire de Joyce Blau publié dans les années 1970 en Turquie, un livre de Kemal Burkay résidant en Suède, quelques livres, dont des rééditions, du poète Cigerxwin alors décédé, une grammaire d'un kurde d'Union Soviétique, une traduction d'Harold Pinter, une histoire du Kurdistan par Ahmet Tîgrîs, enseignant de kurde en Suède. Parmi les publications originales d'ouvrages de personnes résidant alors en Turquie, on trouve uniquement une grammaire de Feqî Hüseyin Sağniç membre de l'Institut Kurde d'Istanbul, un essai historique de Torî. Quelques recueils de poésie sont également publiés.

70 'Li derveyî welêt bêhejmar weşanxaneyên kurdî ava bûn, sazgehên demokratik û kulturî ava bûn, kovar û rojnameyên bi kurdî derketin, filmên bi kurdî çêbûn. Mirov kane bêje ku ronanseke ziman û edebiyata kurdî dest pê kirîbû. Lê, mixabin, ev tevgera kultura kurdî tenê li derveyî welêt dima, ew nedigihîşte hevwelatiyên me yên li welêt. [...] Xebata kulturî ya ku bi gelemperî li Ewrûpayê û bi taybetî li Swedê geşbûbû di dolava xwe de mabû'. Cewerî, Firat 'Edebiyata Kurdî' [La littérature kurde], in Cewerî 1996 : 135. 
Citation: Scalbert Yücel, Clémence (2006) 'La diaspora kurde en Suède. Conservation, production et diffusion d'un savoir linguistique', European Journal of Turkish Studies, Thematic Issue №5, Power, ideology, knowledge - deconstructing Kurdish Studies, URL : http://www.ejts.org/document771.html

To quote a passage, use paragraph (§)

que très peu d'auteurs 'locaux' à publier. La part des rééditions d'ouvrages publiés en

Suède est donc relativement importante les premières années (graphique 2).

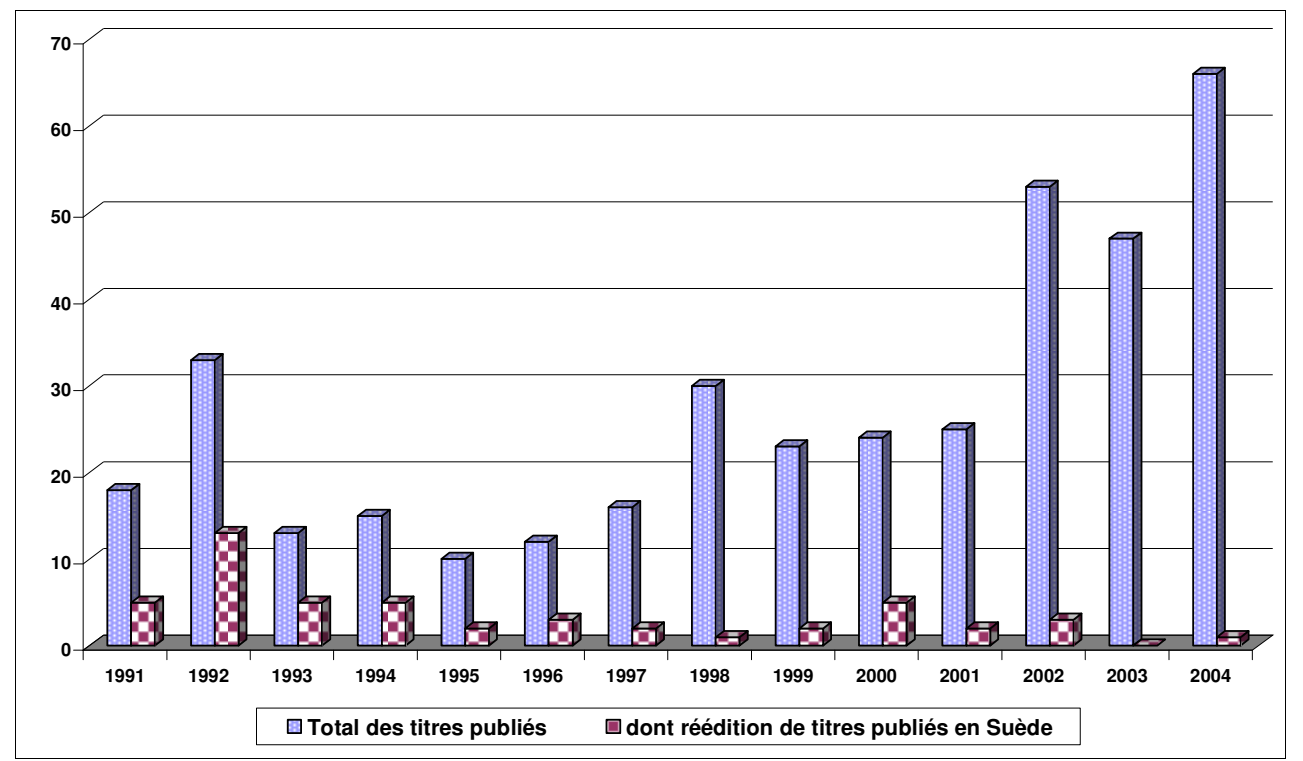

Graphique 2 : Nombre de titres publiés en kurmandji en Turquie et part des rééditions d'ouvrages parus en Suède (1991-2004).

Source : Scalbert-Yücel 2005.

[42] Aujourd'hui, les plus grandes figures de la littérature kurmandji contemporaines sont notamment Mehmet Uzun, Firat Ceweri, Hesenê Metê. Tous résident en Suède, ont écrit dès les années 1980 et ont été réédités en Turquie dans les années 1990-2000. Ils sont aujourd'hui considérés comme la première génération d'auteurs kurdes de Turquie. Ce sont ces auteurs que les lecteurs ont d'abord connus en Turquie ; ce sont avec eux que la nouvelle génération d'écrivains - qui apparaît en Turquie - s'est formée. Le problème de la concurrence intergénérationnelle (doublée d'une concurrence géographique) voit le jour dès lors que, au cours des années 1990, les écrivains kurdes apparaissent en Turquie. L'auteur isolé en Suède doit gérer cette distance au lectorat, distance qui se fait de plus en plus gênante à partir du moment où de nouveaux auteurs apparaissent et sont publiés en Turquie. Ces derniers, s'ils n'ont pas la renommée des auteurs vivant en Suède, appartiennent à un milieu littéraire relativement soudé (intellectuellement et géographiquement) et sont bien plus proches du lectorat potentiel de taille autrement comparable au lectorat suédois. Ainsi, on remarque un retour au pays (définitif ou temporaire, mais dans le dernier cas, régulier) de la vieille génération suédoise. On remarque surtout l'absence de renouvellement générationnel en Suède: quasiment tous les jeunes auteurs sont désormais en Turquie. Enfin, on remarque que les 
Citation: Scalbert Yücel, Clémence (2006) 'La diaspora kurde en Suède. Conservation, production et diffusion d'un savoir linguistique', European Journal of Turkish Studies, Thematic Issue $N^{\circ} 5$, Power, ideology, knowledge - deconstructing Kurdish Studies, URL : http://www.ejts.org/document771.html

To quote a passage, use paragraph $(\S)$

auteurs kurdes en Suède se font de plus en plus souvent éditer directement en Turquie. Et ceci pose clairement la question de la préservation du pôle littéraire et éditorial kurmandji en Suède.

[43] Alors que l'édition en langue kurde se développe en Turquie, à Istanbul ou à Diyarbakir, elle diminue en Suède. Le nombre de titres publiés en Turquie dépasse aujourd'hui celui des titres publiés en Suède dont les chiffres absolus paraissent également diminuer (graphique 1). Parfois même, l'impression est délocalisée : si le siège de l'édition reste en Suède, la maison peut faire imprimer directement en Turquie avec des coûts de revient moins élevés et surtout une proximité avec le lectorat. Le livre est ainsi directement vendu en Turquie. On remarque également le développement d'un travail en binôme avec l'association informelle d'une maison d'édition en Suède et d'une maison en Turquie. Ainsi, dans le domaine de la production littéraire et éditoriale, il semble que le rôle de la diaspora ait été crucial dans la préservation de la langue et dans le développement d'une littérature kurmandji en Turquie même jusque dans les années 1990 mais que ce rôle, alors que les possibles se développent au pays, soit aujourd'hui en recomposition.

\section{Conclusion}

[44] Une immigration kurde fortement politisée conjuguée à des politiques étatiques migratoire et culturelle tout à fait spécifiques en Europe a permis une organisation proprement diasporique de la population kurde en Suède. Du fait de la spécificité des politiques suédoises, c'est essentiellement dans le domaine linguistique et littéraire que s'est illustré ce segment suédois de la diaspora kurde. Ainsi, la langue kurde - comme la culture et l'identité qui lui est associée - a pu y être préservée, normée mais aussi enseignée et diffusée, essentiellement à l'échelle du territoire suédois. La littérature orale a pu être conservée dans les livres, eux-mêmes archivés. L'écriture littéraire a été stimulée et a vu le jour en Suède.

[45] Ces activités de production et de conservation d'un savoir particulier, ces activités de création artistique - souvent indissociable du militantisme - paraissent être d'abord tournées vers la diaspora, fonctionnant en cercle fermé. Pourtant des liens sont clairement tissés entre la diaspora et l'origine, ne serait-ce que par la langue employée, la 
Citation: Scalbert Yücel, Clémence (2006) 'La diaspora kurde en Suède. Conservation, production et diffusion d'un savoir linguistique', European Journal of Turkish Studies, Thematic Issue N5, Power, ideology, knowledge - deconstructing Kurdish Studies, URL : http://www.ejts.org/document771.html

To quote a passage, use paragraph $(\S)$

culture préservée. Des liens concrets se tissent également, surtout dans les années 1990, alors que l'Etat turc devient plus tolérant vis-à-vis de l'emploi de la langue kurde. On voit se créer des réseaux entre associations en diaspora et en Turquie, permettant à la diaspora d'agir concrètement dans le pays d'origine. La diaspora joue ainsi un rôle majeur dans le développement des activités linguistiques en Turquie.

[46] Pourtant, l'amélioration de la situation politique en Turquie qui permet à la diaspora de s'y impliquer concrètement menace aussi son rôle privilégié en matière linguistique. En effet, si la seconde génération, celle des enfants de migrants en Suède, est en général assez impliquée dans la 'cause kurde', elle l'est peu en langue kurde ; les jeunes kurdes, nés et vivant en Suède, deviennent rarement écrivains ou éditeurs, alors que les jeunes écrivains et éditeurs sont de plus nombreux en Turquie. Tant qu'aucune activité n'était possible en Turquie, la diaspora a donc véritablement joué ce rôle de conservatoire et a donné une impulsion qui ne prenait véritablement tout son sens qu'en direction de l'origine. Elle a permis de donner l'impulsion et de guider les plus jeunes générations. Aujourd'hui alors que les conditions commencent à être modifiées dans le pays d'origine, il semble que l'action de la diaspora même puisse être minimisée et que cette diaspora ait à repenser son rôle. 
Citation: Scalbert Yücel, Clémence (2006) 'La diaspora kurde en Suède. Conservation, production et diffusion d'un savoir linguistique', European Journal of Turkish Studies, Thematic Issue №5, Power, ideology, knowledge - deconstructing Kurdish Studies, URL : http://www.ejts.org/document771.html

To quote a passage, use paragraph (§)

\section{Références}

Alakom, Rohat (1991) Di Çavkanîyên Swedî De Motîvên Kurdî [Les motifs kurdes dans les sources suédoises], Stockholm, Vêjîn.

Alakom, Rohat (2002) 'Kurdên Anatoliya Navîn li Swêdê' [Les Kurdes d'Anatolie centrale en Suède], Bîrnebûn 16, pp. 39-58.

Alakom, Rohat (2006) Kurdên Swêdê (1965-2005), Stockholm, Serkland.

Boyd, Sally (2001) 'Immigrant Languages in Sweden', in Extra, G.; Gorter, D. (eds.), The Other Languages of Europe. Demographic, Sociolinguistic and Educational Perspectives, Clevedon, Toronto, Buffalo, Sydney, Multinlingual Matters Limited, pp. 177-192.

Bruneau, Michel (1995) Diasporas, espaces. Mode d'emploi, Montpellier, GIP-Reclus.

Cabau, Béatrice (1996) L'enseignement des langues-cultures en Suède : un enjeu multidimensionnel, Paris, Thèse de Doctorat, Paris III.

Cabau-Lampa, Béatrice (1998) L'enseignement des Langues-Cultures en Suède: un enjeu multidimensionnel, Lille, Presses Universitaires du Septentrion.

Cabau-Lampa, Béatrice (1999) 'Home Language Instruction in Sweden: What is the Situation in 1998?', European Journal of Intercultural Studies 10 (1), pp. 75-84.

Cewerî, Firat (1996) Kultur, Hûner û Edebiyat [Culture, art et littérature], Stockholm, Weşanên Nûdem.

Diljen, Haydar; Erdem, A. J.; Lewendi, Mahmut; Karim, Remzi (2002) Dergûşa Nasnameyê [Le berceau de l'identité], Stockholm, Skolverket.

Dufois, Stéphane (2003) Les diasporas, Paris, Presses Universitaires de France.

Gulyas, Mikos (1977) Kurderna och biblioteken, Lidingö stadsbibliotek.

Gulyas, Mikos (1982) De bloklösa barnen, Statens Invandrarverk.

Haugen E. (1966), 'Dialect, Language, Nation', American Anthropologist 68 (6), pp. 922935.

Ma-Mung, Emmanuel (1994) 'Non-lieu et utopie : la diaspora chinoise et le territoire', Espace Géographique 2, pp. 106-114.

Regeringsproposition (1975) 26 om riktlinjer för invandrar - och minoritetspolitikers.

Regeringsproposition (1975-1976) 118 om hemspråksundervisning för invandrarbarb.

Scalbert, Clémence (2005) 'Kurdes sans Kurdistan', Outre-terre 12 (Dossier : ‘Enseigner la nation, géopolitique des manuels'), pp. 93 - 104.

Scalbert, Clémence (2006) 'L'élaboration de la langue kurde en Turquie (1898 - 1943): d'un simple outil d'éveil national au pivot de la définition identitaire', in Alen-Garabato, Carmen (ed.), L'éveil des nationalités et les revendications linguistiques en Europe (18301930), Paris, L'Harmattan, pp. 255-274.

Scalbert Yücel, Clémence (2005) Conflit linguistique et champ littéraire kurde en Turquie, Thèse de doctorat, sous la direction de Michel Korinman, Université Paris IV, Paris. 
Citation: Scalbert Yücel, Clémence (2006) 'La diaspora kurde en Suède. Conservation, production et diffusion d'un savoir linguistique', European Journal of Turkish Studies, Thematic Issue N5, Power, ideology, knowledge - deconstructing Kurdish Studies, URL : http://www.ejts.org/document771.html

To quote a passage, use paragraph $(\S)$

Simon, Gabriel (1995), Géodynamiques des migrations internationales dans le monde, Paris, Presses Universitaires de France.

SOU (1974) Invandrarutredningen 3 C Invandrarna och minoriteterna, SOU 1974, 69\&70, Stockholm, Utbildningsförlaget.

Tayfun, Mehmet (1998) Kurdiskt författarskap och kurdisk bokutgivning. bakgrund, villkor, betydelse [Écriture et édition kurdes - Aperçu historique, conditions, significations], Stockholm, Apec.

Thiesse, Anne-Marie (1999) La création des identités nationales en Europe XVIllèmeXXème siècles, Paris, Le Seuil.

Uzun, Mehmet (1996) Ziman û Roman [La langue et le roman], Stockholm, Nûjen. 